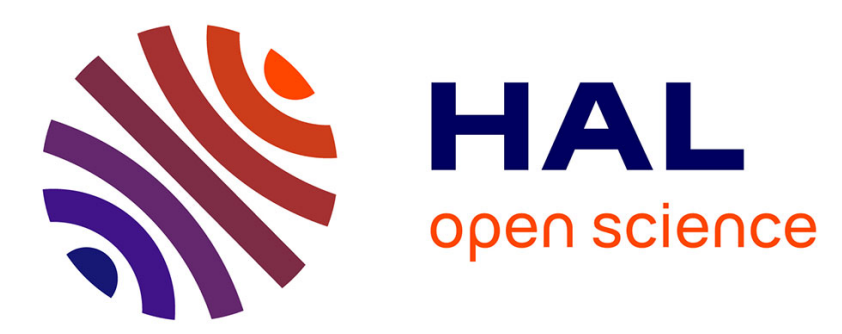

\title{
Multiobjective optimization for the design of phase III biorefinery sustainable supply chain
}

\author{
Andrea Teresa Espinoza Pérez, Paulo César Narváez Rincón, Mauricio
}

Camargo, Miguel Domingo Alfaro Marchant

\section{To cite this version:}

Andrea Teresa Espinoza Pérez, Paulo César Narváez Rincón, Mauricio Camargo, Miguel Domingo Alfaro Marchant. Multiobjective optimization for the design of phase III biorefinery sustainable supply chain. Journal of Cleaner Production, 2019, 223, pp.189-213. 10.1016/j.jclepro.2019.02.268 . hal02072187

\section{HAL Id: hal-02072187 \\ https://hal.science/hal-02072187}

Submitted on 29 Dec 2021

HAL is a multi-disciplinary open access archive for the deposit and dissemination of scientific research documents, whether they are published or not. The documents may come from teaching and research institutions in France or abroad, or from public or private research centers.
L'archive ouverte pluridisciplinaire HAL, est destinée au dépôt et à la diffusion de documents scientifiques de niveau recherche, publiés ou non, émanant des établissements d'enseignement et de recherche français ou étrangers, des laboratoires publics ou privés. 


\title{
Multiobjective optimization for the design of Phase III Biorefinery sustainable supply chain
}

\author{
Andrea Teresa Espinoza Perez ${ }^{\text {a,c }}$, *, Paulo Cesar Narvaez Rincon ${ }^{\mathrm{b}}$, Mauricio Camargo ${ }^{\mathrm{c}}$, \\ Miguel Domingo Alfaro Marchant ${ }^{\text {a }}$ \\ a Departamento de Ingeniería Industrial, Universidad de Santiago de Chile, Av. Ecuador, 3769, \\ Santiago, Chile \\ b Departamento de Ingeniería Química y Ambiental, Grupo de Procesos Químicos y \\ Bioquímicos, Facultad de Ingeniería, Universidad Nacional de Colombia. Sede Bogota, Carrera \\ 30 45-03, Edificio 412, Bogota, Colombia
}

c Universite de Lorraine, ERPI, F-54010, France

Please cite as :

Pérez, A. T. E., Rincón, P. C. N., Camargo, M., \& Marchant, M. D. A. (2019). Multiobjective optimization for the design of phase III biorefinery sustainable supply chain. Journal of Cleaner Production. Volume 223, 20 June 2019, Pages 189-213 https://doi.org/10.1016/j.jclepro.2019.02.268

\begin{abstract}
Bioeconomy has gained importance during the last decade as a frame of reference for the design and implementation of policies to foster sustainable development and innovation. Considering the need for society to move to forms of production in which generation of wastes and the use of fossil fuels have to be minimized or eliminated, Phase III biorefineries (mix feedstock and multiple products) have emerged as a promising and central element in bioeconomy. However its industrial development has been slower than expected, especially due to investors' poor perception of technological risk and large investment required. Among the current climate change challenges, the need of methodologies and tools to develop an optimal supply chain for the biorefinery, considering all the aspects of sustainability and taking into account the territorial context of the project is fundamental. The present work proposes a comprehensive decisionmaking approach for a Phase III biorefinery project, by integrating all the sustainability dimensions. This approach is formalized and solved thanks to a multiobjective optimization model, where sustainability criteria are included in the formulation of objective functions, decision variables and constraints. In order to illustrate the proposed approach, a case study was developed for the Colombian context. The resulting Pareto fronts shown that biorefineries integrating different types of raw materials, even non-food crops, are economically feasible in North Colombia.
\end{abstract}




\section{Introduction.}

Bioeconomy has gained importance during the last decade as a frame of reference for the design and implementation of policies to foster sustainable development and innovation. Nowadays, given the urgent need of our society to move toward forms of production in which the generation of waste and the use of fossil fuels are minimized or eliminated (Rodríguez et al., 2017), biorefineries have emerged as a promise and central element in bioeconomy. A biorefinery is a particular project that must be considered in its territorial context as a part of a sustainable development strategy. Indeed, it concerns at the same time, the valorization of a local available biomass and the integration of a new industrial activity within the targeted territory (Ceapraz et al., 2016). From a territorial development point of view, some of the objectives of a biorefinery are (i) support energy supply security and reduce dependency on oil imports (ii) support rural areas development through technology deployment and creation of jobs, and (iii) mitigate the greenhouse gas (GHG) - promoting a low carbon and sustainable economy (Bautista et al., 2016; Höltinger et al., 2014; Sammons et al., 2007; Sukumara et al., 2013; Valdivia et al., 2016).

However, the development of biorefineries has been slower than expected, especially when nonfood crops are employed as feedstocks. In 2016, among the 423 existing biorefineries worldwide, only 84 were using non-food feedstock. Additionally, only a third of these (26) were operating on commercial scale (Nguyen et al., 2017). This low implementation can be explained by the perception of the investors who believe that this type of project implies highly technological risk with low profitability, due to a lack of well-defined logistical models related to the raw material collection, transport and storage, as well as the requirement of the development of new technologies to transform biomass in the desired products (Bautista Rodríguez, 2015; Valdivia et al., 2016).

Furthermore, any type of biorefinery project at industrial scale needs large investments. The median oil-to-biodiesel plant has a capital expenditure of 465 USD per ton. In the case of dry corn mill ethanol plant the capital expenditure is 757 USD per ton, whereas it is 2899 USD per ton for a lignocellulosic and 3042 USD per ton for a thermochemical ethanol production biorefinery (Tsagkari, M., Couturier, J., Kokossis, A., \& Dubois, 2016). Therefore, trustable studies providing information related to project constraints and opportunities, technology to be used, structural and labor requirements, are needed in order to reasonably decide whether the project could be implemented or not. The traditional feasibility assessments are focused on the evaluation of the technical and economic performances of the project. However, this type of evaluation alone is not always enough to estimate accurately the potential biorefinery project performance; due to other considerations that could eventually limit the biorefinery development. For example, global biomass availability is heterogeneous and limited, which leads to the potential competition for biomass between different biomass consuming sectors. In addition, while certain biomass sources, such as forestry and fisheries are naturally renewed within short periods, they can be used to exhaustion depending on the degree and type of exploitation (Ronzon et al., 2017).

In order to avoid the exhaustion of natural resources ensuring the attainment and continued satisfaction of human needs for present and future generations, a number of factors have to be considered. Including the need for maintaining life support systems such as water services, essential biodiversity needs and potential competition for land between different sectors of primary biomass production, among others (Ronzon et al., 2017). The performance evaluation that integrates these aspects is known as sustainability assessment (Bautista et al., 2016; Global Reporting Initiative, 2016).

A recent literature review (Espinoza Pérez et al., 2017b) shown that very few researches have considered the whole sustainability dimensions when designing the strategic planning of biorefineries in a territory. So, it can be concluded that, although the growing scientific literature in this field sustainability considerations on this type of studies still partial. Because the objectives sought were defined without the use of a sustainability guide in in the set of analyzed researches. 
Thus, there is a need of methodologies and tools to develop an optimal supply chain (SC) for the biorefinery, considering all the aspects of sustainability and taking into account the territorial context of the project. This research aims to fill this gap by developing an optimization model to design a biorefinery integrating the sustainability assessment, based on two sustainability assessment guides, and the SC decisions from feedstock supply to the product distribution and consumption. Besides it, considers multi biomass sources to produce multiple types of biobased products, by different production technology options, i.e. a Phase III biorefinery. To achieve this aim, a multi-objective mixed integer non-linear programming model is developed and the set of optimal solutions, obtained through an evolutionary algorithm is used. In addition, computational experiments are performed on a case study aiming to illustrate the applicability of the proposed model: the biodiesel and biopolymers production from alternative oils in Colombia -. Finally, sensitivity analyses are conducted to provide deeper understanding of the proposed model and the case study.

This article is organized as follows. Section 2 provides a literature review about biorefinery SC researches. Section 3 describes the problem, as well as the optimization models and the solution approach. In Section 4, the case study is described and the proposed model is applied. Additionally, results are presented, analyzed and discussed. Finally, in Section 5 the conclusions along future research directions are promulgated.

\section{Literature Review}

Among the different challenges that have to be overcome for biorefinery implementation, a sustainable well designed and well-managed SC is a key condition (Ekşioğlu et al., 2009). Thus, in order to found the literature related to biorefinery SC, a search strategy was designed including the following steps:

(1) Defining keywords to perform the search in databases: "Supply chain" and "Biorefinery";

(2) Establishing sources of information to be employed (databases): "Web of Science" and "Scopus";

(3) Delimiting the period to be explored: 01 January 2016 to 10 September 2018, in order to complete the literature review carried out in a previous work (Espinoza Pérez et al., 2017b).;

(4) Making an initial selection of documents.

According to the search strategy described above, 126 scientific publications were found. Among which, 102 investigations are surrounding to biorefinery SC. These include 26 documents related to literature reviews for the biorefinery $\mathrm{SC}$, the biorefinery production technologies and the biomass production subjects. In addition, 15 research documents present different assessments for biorefineries (techno-economic assessment) and five investigations focus only in the production process optimization. Finally, 56 articles related to the biorefinery supply chain design using optimization and/or simulation approaches were found for the designed period.

\subsection{Previous literature reviews}

Since 2016, several bibliographic reviews related to biorefineries have been carried out. As can be seen in Figure 1, most of these reviews have focused on supply chains and sustainability. In addition, as can be seen in the detail presented in Table 1, there are three investigations that have reviewed different ways of obtaining raw materials, various production technologies for biorefineries, along with the elements that should be considered in the design of supply chains and sustainability (Black et al., 2016; Özdenkçi et al., 2017; Shastri, 2017).

The research presented by Black et al., (2016) address the development of a comprehensive database to support the business community to develop biomass supply chains and conversion decision-making. The database covers origin, logistics, technical feasibility and policy. (Renewable energy, bioenergy) analyze lignocellulosic and microalgal biomass, finding that for lignocellulosic biofuels the development of efficient pretreatment methods and improved enzymes is a key challenge. In the case of microalgal biofuels, increasing culture density and reducing dewatering requirements needs immediate efforts. In addition, practical biorefinery 
implementation, scale-up and sustainability-related challenges are presented. In the research presented by (A novel biorefinery integration concept for lignocellulosic biomass) a biorefinery for lignocellulosic biomass is proposed, considering a sectoral integration network and a new hydrothermal process for biomass conversion. The sectoral integration concept considers pretreatment at the biomass sites, regional distributed conversion of biomass from various sectors and centralized upgrading/separation of crude biofuels. On the other hand, the conversion technology proposed is compared to other transformation processes.

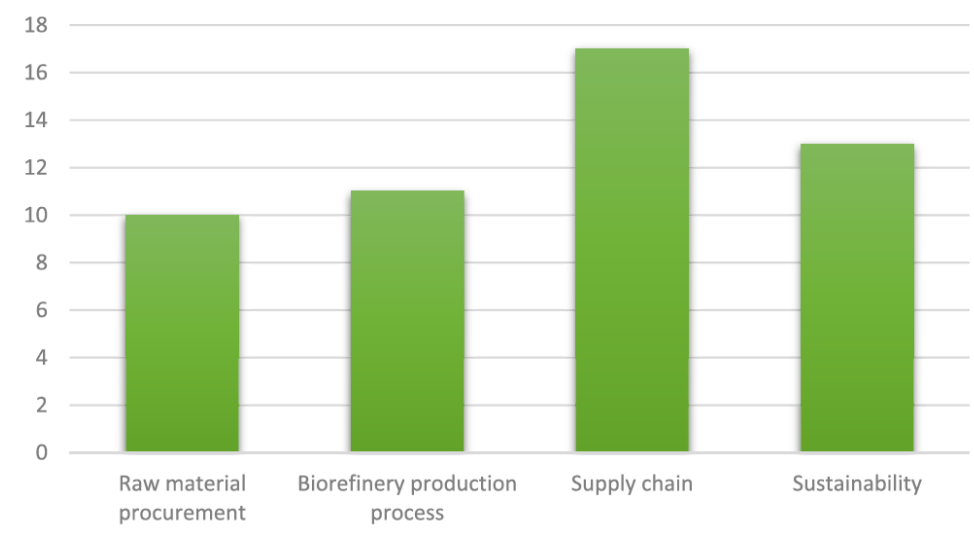

Figure 1. Literature review subject

Among the several investigations devoted to biorefinery sustainable SC (SSC), a common conclusion is the need for an integrated biorefinery model considering multiple perspectives, policy uncertainty and a diversified final product portfolio with new high-value bio-based materials and chemicals. In addition, this model should address the optimization of transformation and biomass acquisition costs. Among the different optimization criteria found, the preferred ones for single-objective optimization are the minimization of total cost, the maximization of total profit and the maximization of net present value. Furthermore, sustainability has been mainly tackled by assessing economic and environmental aspects, leaving behind the social aspects. It means, there is a lack of a clear definition of sustainability. As conclusion, there is still the challenge to strengthen the incorporation of sustainability in the design of biorefinery projects, considering the context of the project.

Table 1. Previous literature review related to biorefinery

\begin{tabular}{|c|c|c|c|c|}
\hline Review & $\begin{array}{l}\text { Raw material } \\
\text { procurement }\end{array}$ & $\begin{array}{c}\text { Biorefinery } \\
\text { production process }\end{array}$ & $\begin{array}{l}\text { Supply } \\
\text { chain }\end{array}$ & Sustainability \\
\hline (Hong et al., 2016) & & & X & $\mathrm{X}$ \\
\hline (Adekunle et al., 2016) & $\mathrm{X}$ & & & \\
\hline (Budzianowski and Postawa, 2016) & & & $\mathrm{X}$ & $\mathrm{X}$ \\
\hline (García et al., 2016) & $\mathrm{X}$ & & $\mathrm{X}$ & \\
\hline (Golecha and Gan, 2016a) & $\mathrm{X}$ & $\mathrm{X}$ & $\mathrm{X}$ & \\
\hline (Jardine and Sayed, 2016) & $\mathrm{X}$ & & & \\
\hline (Matharu et al., 2016) & & $\mathrm{X}$ & & \\
\hline (Black et al., 2016) & $\mathrm{X}$ & $\mathrm{X}$ & $\mathrm{X}$ & $\mathrm{X}$ \\
\hline (Zandi Atashbar et al., 2017) & & & $\mathrm{X}$ & $\mathrm{X}$ \\
\hline (Shastri, 2017) & $\mathrm{X}$ & $\mathrm{X}$ & $\mathrm{X}$ & $\mathrm{X}$ \\
\hline (Chen and Smith, 2017) & & & $\mathrm{X}$ & \\
\hline (Dessbesell et al., 2017) & $\mathrm{X}$ & $\mathrm{X}$ & $\mathrm{X}$ & \\
\hline (Espinoza Pérez et al., 2017b) & & & $\mathrm{X}$ & $\mathrm{X}$ \\
\hline (Maina et al., 2017) & & $\mathrm{X}$ & & $\mathrm{X}$ \\
\hline (Palmeros Parada et al., 2017) & & & $\mathrm{X}$ & $\mathrm{X}$ \\
\hline (Husgafvel et al., 2017) & & & $\mathrm{X}$ & $\mathrm{X}$ \\
\hline
\end{tabular}




\begin{tabular}{|c|c|c|c|c|}
\hline (Özdenkçi et al., 2017) & $\mathrm{X}$ & $\mathrm{X}$ & $\mathrm{X}$ & $\mathrm{X}$ \\
\hline (Shah et al., 2017) & $\mathrm{X}$ & & & \\
\hline (Benjamin et al., 2017) & & $\mathrm{X}$ & & \\
\hline (Chandel et al., 2018) & & $\mathrm{X}$ & & \\
\hline (Barbosa-Póvoa et al., 2018) & & & $\mathrm{X}$ & $\mathrm{X}$ \\
\hline (Zabaniotou, 2018) & & & $\mathrm{X}$ & $\mathrm{X}$ \\
\hline (Parada et al., 2018) & & & $\mathrm{X}$ & $\mathrm{X}$ \\
\hline (Almena et al., 2018) & & $\mathrm{X}$ & & \\
\hline (Esteban and Ladero, 2018) & & $\mathrm{X}$ & & \\
\hline (Srai et al., 2018) & $\mathrm{X}$ & & $\mathrm{X}$ & \\
\hline
\end{tabular}

\subsection{Biorefinery supply chain design using optimization and/or simulation approaches}

The 56 articles related to the biorefinery SC design using optimization and/or simulation approaches were integrated with those presented on our previous work Espinoza Pérez et al., (2017b), reaching 131 documents between 01 January 2006 and 10 September 2018. The increasing trend in this research field can be noted in Figure 2.

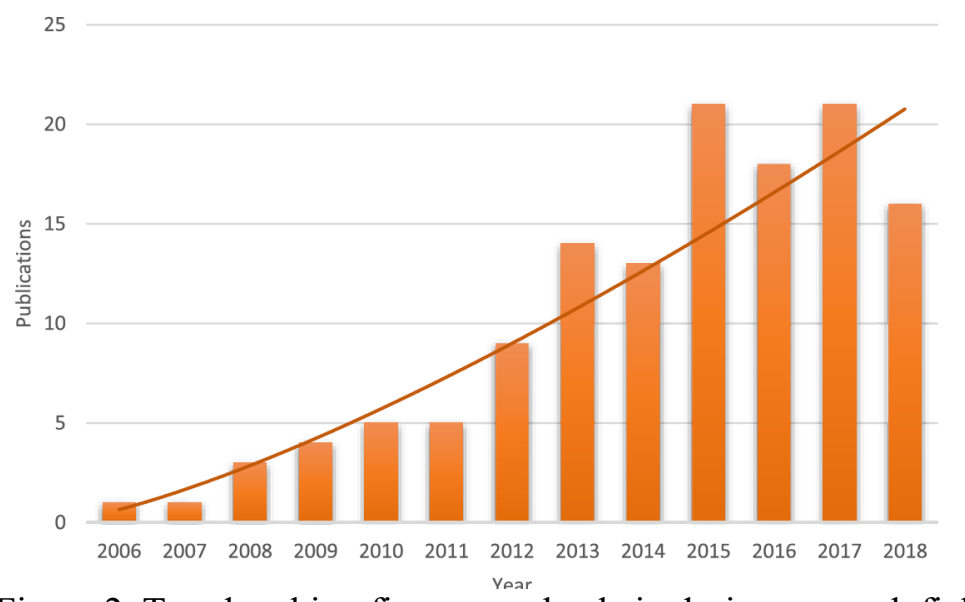

Figure 2. Trend on biorefinery supply chain design research field

Those 131 documents were analyzed in terms of: the sustainability aspect included, the type of model developed (Optimization, simulation or both), the inclusion of uncertainty in the model and the SC decision-making levels integrated in the research.

The sustainability aspects assessed are classified according to the hierarchy described by Bautista et al., (2016), which considers five dimensions of sustainability: economic, social, environmental, technological and political. Because this new concept, named TBL+ (Triple Bottom Line Plus), is highly detailed in terms of quantifiable indicators. Furthermore, the integration of technological and political dimensions is essential for companies such as biorefineries. Because, related to the political dimension, governmental policies are crucial for promoting its implementation, creating economic conditions and favorable markets through subsidies, tax exemptions, and mandatory consumption as in the case of fuel-biofuel mixture (Bautista et al., 2016). Moreover, the technological dimension is also important, considering totally new or adapted technologies are required to transform the raw material into new products, and there are concerns about technological learning, royalties or technology substitution among other aspects related to new products and processes (Bautista et al., 2016).

The SC decision-making levels are classified under three decision perspectives: strategic, tactical and operational (Mortazavi et al., 2015). The strategic level covers long-term decisions, as target markets selection, the plant location, capacity and production technology, biomass selection type 
and location (Chopra and Meindl, 2012; Majid Eskandarpour, 2015; De Meyer et al., 2014). While the tactical level includes the management of medium-term decisions, including inventory planning, biomass harvest planning, storage sites selection, transport mode selection and shipment sizing (Awudu and Zhang, 2012; Guillén et al., 2006). The operational level corresponds to short-term decisions, which concern to inventory planning (daily inventory control, lack of inventory at distribution points) and to programming vehicles (Tsolakis et al., 2014)

Figure 3 presents the distribution of the reviewed publications according to their scope (Economic, Environmental, Social, Technological or Political), the applied approach (simulation and/or optimization) and decision-making levels in SC studied (strategic, tactical or operational). From the figure, it appears that studies focused exclusively on economic objectives are the most common (43) and they mostly deal with optimization. On the opposite side, the political dimension of sustainability is the least studied, with only seven publications that included government incentives. Furthermore, 36\% of the publications include the three decision-making levels. The classification in detail is presented in the Appendix A.

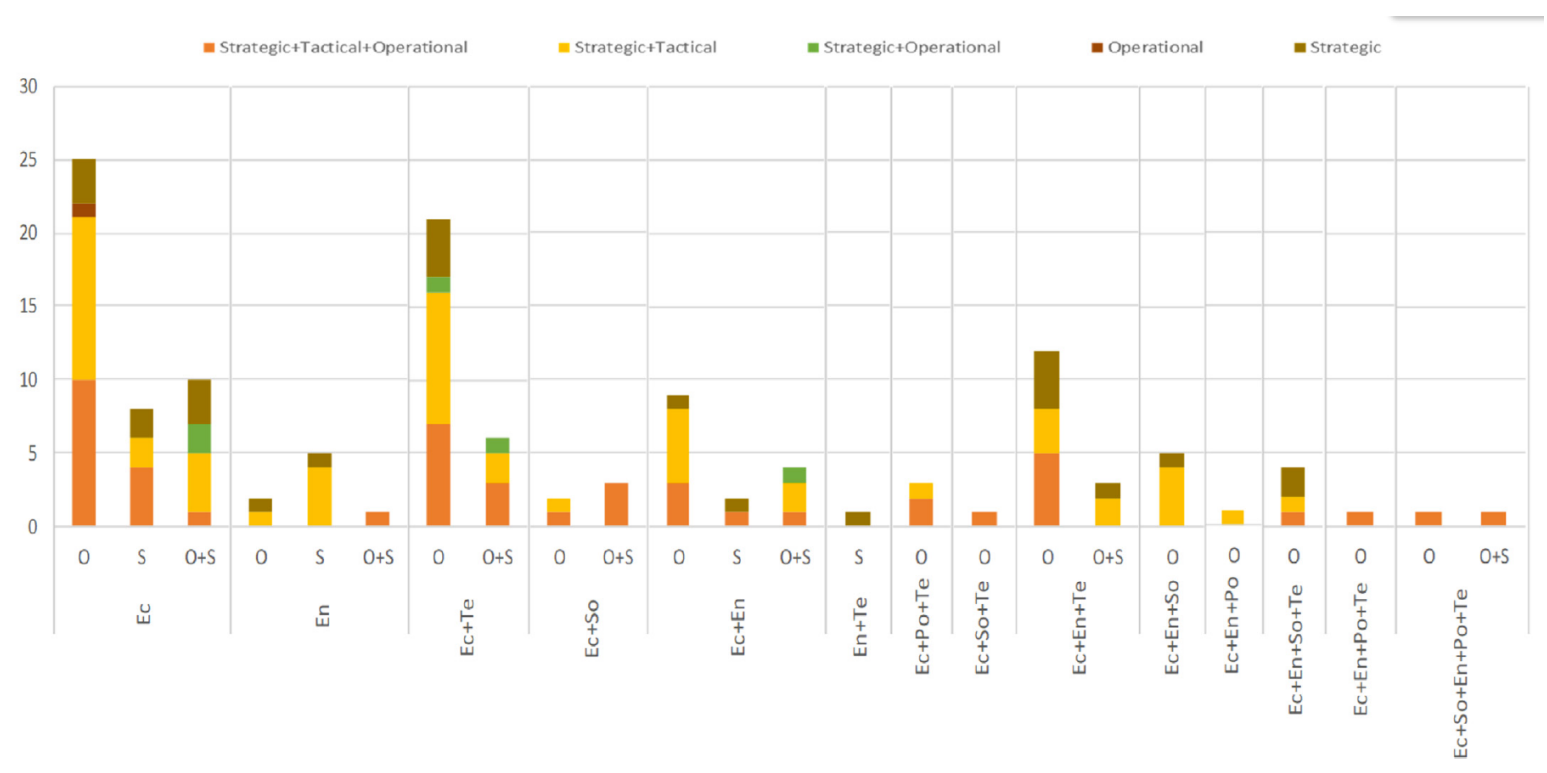

Figure 3. Publication distribution according to the dimensions of sustainability $(\mathrm{Ec}=$ Economic, $\mathrm{En}=$ Environmental, $\mathrm{So}=$ Social, $\mathrm{Po}=$ Political, $\mathrm{Te}=$ Technological $)$ objective $(\mathrm{O}=$ Optimization, $\mathrm{S}=$ Simulation) and decision level studied

It can be concluded, observing figure 3, that the challenges presented in the bibliographic reviews of section 2.1. remain unresolved. Because, even though two studies take into consideration the five dimensions of sustainability, You et al., (2012) and Yue et al., (2014), they do not cover the whole sustainability aspect of each dimension. The first research uses ASPEN to simulate different possible production lines to choose the production technology and includes government incentives as incomes. Its objective is to minimize the annualized costs, maximize local job creation and minimize greenhouse gas emissions. On the other hand, Yue et al., (2014) evaluate the cost of producing electricity, the number of local jobs created and the environmental impacts associated with the production of a unit of bioelectricity, by LCA methodology. It also considers government subsidies as income for the biorefinery. In both studies only one production technology per plant could be chosen and no consideration is given to economies of scale in the technological dimension of sustainability. None of these last studies evaluates the target market selection. 
From the above evidence, it is clear that the biorefinery SSC is still studied in a fragmented and partial manner. Therefore, due to the growing importance of bioeconomy, decision-making approaches and tools are needed to develop and implement these projects. Thus, in order to addressee these gaps in literature and to analyze the potential benefits and untoward effects of Phase III biorefineries, this paper proposes a new multiobjective optimization model to design the strategical decisions in a Phase III Biorefinery SSC. The major contributions and novelties of this study are:

1. The developed model optimizes the strategic decision-making level of the SC configuration for the Phase III Biorefinery, covering multiple types of: feedstock, conversion technologies, production capacity, preprocessing process and biobased products.

2. The model development was based on the analysis of two detailed sustainability frameworks. In order to define sustainable objective functions, constraints and decision variables.

\section{Problem description and model formulation \\ 3.1. Problem description}

This paper focuses on designing an optimized SSC for biomass-based production, able to respond rapidly to changes in the market and suppliers environment. Moreover, the strategic decisionmaking level on SC is the most important to assess in a biorefinery project, in terms of large investments amounts required, long-term decisions and uncertainty. Thus, this level should be the first to be integrated in the optimization model.

Therefore, the SC network for a Phase III Biorefinery integrated the following elements:

1. Biomass source sites to supply multiple types of feedstock

2. Pretreatment facilities processing biomass to accomplish better yields in principal transformation process assessing several potential production technologies and capacities

3. Conversion plants assessing several potential production technologies and capacities

4. Demand nodes for multiple types of biobased products produced in the pre-processing and conversion facilities

In addition, due the nature of this research related to biobased products and biofuels, the sustainability assessment classification in five dimensions has been selected (Bautista et al., 2016). Consequently, the GRI aspects were analyzed according to these five dimensions as outlined below:

Economic. The main economic objective is to design a self-sustaining industry. It will not need government assistance or reinvestments, because it will have the necessary profitability to be self-sustaining (Cambridge Dictionary Online, 2015). Several metrics can be used to measure this objective, as presented for example in Barbosa-Póvoa et al., (2018) and Espinoza Pérez et al., (2017b).

Social. Regarding the social dimension, studies conducted on Biorefinery SSC have measured two edges: the first is related to employment generation and the second to social welfare in terms of food security (Bai et al., 2012; Singh et al., 2014). However, the topics considered in the social dimension must also include respect for property land rights, social acceptability, and promotion of responsible working conditions (Bautista et al., 2016).

Environmental. Among various approaches, life cycle assessment (LCA) is the one most used in studies that consider environmental impact (Yue et al., 2014b). Environmental principles considered in this dimension can be analyzed in regard to issues such as air, soil and water quality, waste and wastewater management, balance of greenhouse gases, conservation and protection of biodiversity and energy efficiency (Ruiz-mercado et al., 2012). 
Technological. This dimension refers to the available production technologies and its readiness level, as well as its evolution through technological learning based on production (de Wit et al., 2010). It also takes into account technological trends in products use and production.

Political. It refers to promotion or restriction policies that may be promulgated by governments or multilateral organizations, as well as possible subsidies and tax reductions to stimulate the market (Bautista et al., 2016).

Furthermore, the process of modelling the strategic decision-making level of Phase III Biorefinery SC and the sustainability aspects can be considered as "art", because there are no consigns about how to build a model (Espinoza Pérez et al., 2017a).

As previously mentioned in section 2 , most of the investigations integrate all the decision-making levels in SC but not all the aspects of sustainability where addressed. Thus, in order to consider all the aspects, a strategy to deal with the complex task of creating the optimization model is presented (Figure 4). The objective is to integrate the $\mathrm{SC}$ decision-making level components at first (horizontal axis); in order to analyze each sustainability dimension subsequently, one at a time (vertical axis). This strategy permits a better understanding of the model and the possibility of test it at each addition of components.

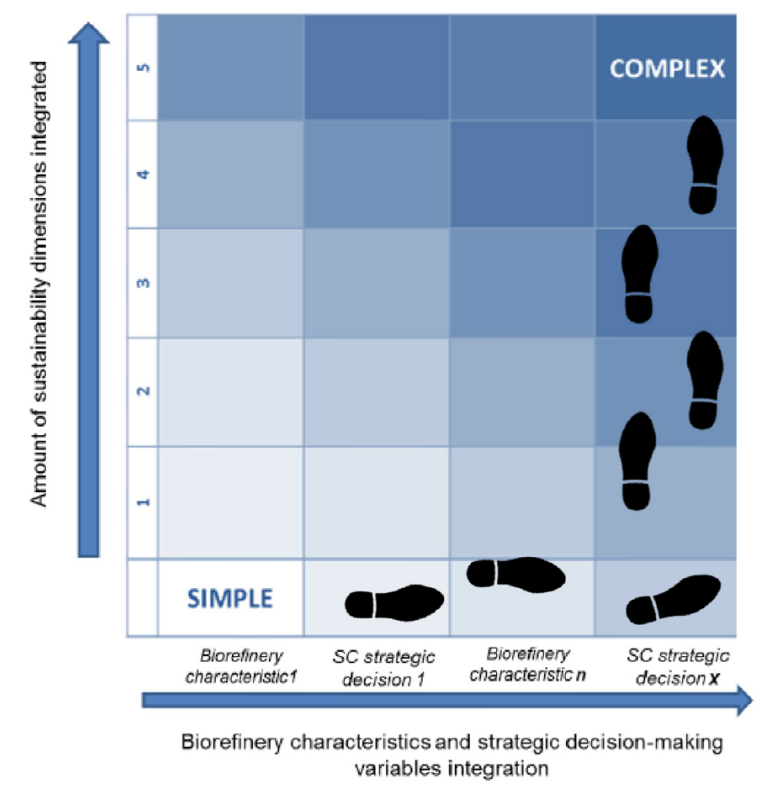

Figure 4. Modelling strategy for consider the five dimensions of sustainability in a Phase III Biorefinery SSC

\subsection{Model Formulation}

\section{Model contraints}

\section{Mass balances}

Eq. (1)

$$
\sum_{c} \sum_{n} \sum_{f} \alpha_{n, c, b}\left[\sum_{i} N_{i, n, j, c, f}\right]=\sum_{k} \sum_{\substack{d \\ \forall j, b}} \sum_{g} R_{j, b, k, d, g}+\sum_{c} R e c_{b, j, c}+\sum_{m} T_{j, b, m}
$$


Eq. (2)

$$
\sum_{d} \sum_{b} \sum_{g} \alpha_{b, d, a} *\left[\sum_{j} R_{j, b, k, d, g}\right]=\sum_{l} P_{k, a, l}+\sum_{d} R e u_{a, k, d}
$$

Raw materials availability

Eq. (3)

$$
\begin{gathered}
\sum_{j} \sum_{c} \sum_{f} N_{i, n, j, c, f} \leq \text { RMAvailavility }_{i, n} \\
\forall i, n
\end{gathered}
$$

Processing capacity

Eq. (4)

$$
\begin{gathered}
\sum_{n} \sum_{i} N_{i, n, j, c, f} \leq \operatorname{CapPP}_{j, c, f} * P P_{j, c, f} \\
\forall j, c, f
\end{gathered}
$$

Eq. (5)

$$
\begin{gathered}
\sum_{b} \sum_{j} R_{j, b, k, d, g} \leq \operatorname{Cap}_{k, d, g} * W_{k, d, g} \\
\forall k, d, g
\end{gathered}
$$

Production capacity and technology by plant

Eq. (6)

$$
\sum_{c} \sum_{f} P P_{j, c, f} \leq 1
$$

$\forall j$

Eq. (7)

$$
\sum_{g} \sum_{d} W_{k, d, g} \leq 1
$$

Maximum demand

Eq. (8)

$$
\begin{gathered}
\sum_{j} T_{j, b, m} \leq \operatorname{Dem}_{m, b}(\operatorname{Max}) \\
\forall m, b
\end{gathered}
$$

Eq. (9)

$$
\begin{gathered}
\sum_{k} P_{k, a, l} \leq \operatorname{Dem}_{l, a}(\operatorname{Max}) \\
\forall l, a
\end{gathered}
$$

\section{Auxiliary constraints}

Eq. (10)

$$
\sum_{b} \sum_{m} T_{j, b, m} \leq M * \sum_{c} \sum_{f} P P_{j, c, f}
$$

Eq. (11) 


$$
\sum_{b} \sum_{k} \sum_{d} \sum_{g} R_{j, b, k, d, g} \leq M * \sum_{c} \sum_{f} P P_{j, c, f}
$$

Eq. (12)

$$
\sum_{a} \sum_{l} P_{k, a, l} \leq M * \sum_{d} \sum_{g} W_{k, d, g}
$$

\section{Governmental incentives constraint for plants installation}

Eq. (13)

$$
\sum_{j} I n c P_{j}+\sum_{k} I n c W_{k} \leq \text { LimBudget }
$$

Eq. (14)

$$
\operatorname{IncP}_{j} \leq \operatorname{Min}\left\{\operatorname{incCap}_{j}\left[\sum_{c} \sum_{f} P P_{j, c, f}\right] ; \operatorname{incPerP}_{j}\left[\sum_{c} \sum_{f} \operatorname{Inv}_{j, c, f}\right]\right\}
$$

Eq. (15)

$$
\operatorname{IncW}_{k} \leq \operatorname{Min}\left\{\operatorname{incCap}_{k}\left[\sum_{d} \sum_{g} W_{k, d, g}\right] ; \operatorname{incPer} W_{k}\left[\sum_{d} \sum_{g} \operatorname{Inv} v_{k, d, g}\right]\right\}
$$

Governmental incentives constraint for production technologies

Eq. (16)

$$
\left(\sum_{c} \text { InvTechP } P_{c}+\sum_{d} \text { InvTechW } W_{d}\right) \leq \text { BudProjValueTech }
$$

Governmental investments constraint for electric infrastructure

Eq. (17)

$$
\begin{gathered}
\operatorname{Dem}_{a_{E}, l}^{\prime}=\operatorname{Dem}_{a_{E}, l}+(\xi * \operatorname{GovInvE}) \\
\forall a_{E}, l
\end{gathered}
$$

Water availability

Eq. (18)

$$
\begin{aligned}
\left\{\sum_{n} \text { WRawMat }_{n}\right. & * \sum_{i} \sum_{j} \sum_{c} \sum_{f} N_{i, n, j, c, f}+\sum_{n} \sum_{c} \sum_{f}\left(\text { WPret }_{n, c, f} *\left[\sum_{i} \sum_{j} N_{i, n, j, c, f}\right]\right) \\
& \left.+\sum_{b} \sum_{d} \sum_{g}\left(\text { WPlant }_{b, d, g} *\left[\sum_{j} \sum_{k} R_{j, b, k, d, g}\right]\right)\right\} \leq \text { TotalWaterAval }
\end{aligned}
$$

\section{Model objective functions}

Maximization of the installed capacity use normalized

Eq. (19)

$$
\operatorname{Max}\left\{\frac{\left[\frac{\sum_{j} \sum_{c} \sum_{f}\left(\sum_{i} \sum_{n} N_{i, n, j, c, f}\right)}{\sum_{j} \sum_{c} \sum_{f} \operatorname{CapPP} P_{j, c, f} * P P_{j, c, f}}+\frac{\sum_{k} \sum_{d} \sum_{g}\left(\sum_{j} \sum_{b} R_{j, b, k, d, g}\right)}{\sum_{k} \sum_{d} \sum_{g} \operatorname{CapW}_{k, d, g} * W_{k, d, g}}\right]}{2}\right\}
$$


Maximization of the net present value

Eq. (20)

$$
\operatorname{Max} \sum_{y=1}^{T} \frac{\text { Net } \text { Cash Flow }_{y}}{(1+\text { Discount Rate })^{y}}-\text { Initial Investment }+\left(\sum_{j} \text { IncP }_{j}+\sum_{k} \text { IncW }_{k}\right)
$$

Where, in order to facilitate the calculation, it is assumed that the cash flows will be the same during the time of evaluation of the net present value. Thus, the expression for cash flows can be represented by:

$$
\text { Net Cash Flow } y=y *(\text { Incomes }- \text { Costs })
$$

Incomes can be described as the products sold in the market by their market value, plus the products that are re-used in the production plants by their value:

$$
\begin{aligned}
\text { Incomes }=\sum_{a} \operatorname{Prix}_{a} *\left[\sum_{k} \sum_{l} P_{k, a, l}\right]+\sum_{b} \operatorname{Prix}_{b} *\left[\sum_{j} \sum_{m} T_{j, b, m}\right]+\sum_{b} \sum_{j} \operatorname{val}_{b, j} *\left[\sum_{c} \operatorname{Rec}_{b, j, j, c}\right] \\
+\sum_{a} \sum_{k} \operatorname{val}_{a, k} *\left[\sum_{d} \operatorname{Reu}_{a, k, k, d}\right]
\end{aligned}
$$

Besides, Costs are represented by transportation cost, raw material acquisition cost, transformation cost and the pollution cost.

The transportation costs consist on the cost of moving the raw materials to pretreatment plants; transport the intermediate products to their respective markets and / or the main production plants, and the transport of the final products to the markets:

$$
\begin{aligned}
\text { TransportCost } & =\sum_{i} \sum_{j} \operatorname{TCost}_{i, j} *\left[\sum_{c} \sum_{f} \sum_{n} N_{i, n, j, c, f}\right]+\sum_{j} \sum_{k} \operatorname{TCost}_{j, k} *\left[\sum_{d} \sum_{g} \sum_{b} R_{j, b, k, d, g}\right] \\
& +\sum_{k} \sum_{l} \operatorname{TCost}_{k, l} *\left[\sum_{a} P_{k, a, l}\right]+\sum_{j} \sum_{m} \operatorname{TCost}_{j, m} *\left[\sum_{b} T_{j, b, m}\right]
\end{aligned}
$$

The raw material acquisition cost depends on raw material price:

$$
\text { RawMaterialsCost }=\sum_{n} \text { RMCost }_{n} *\left[\sum_{i} \sum_{j} \sum_{c} \sum_{f} N_{i, n, j, c, f}\right]
$$

The costs of production or operation depend on the incoming materials type, the transformation technology used, the production capacity and the plant location:

$$
\begin{aligned}
\text { ProdCost }=\sum_{n} \sum_{j} \sum_{c} \sum_{f} \text { Post }_{n, j, c, f} *\left(\sum_{i} N_{i, n, j, c, f}\right) \\
+\sum_{b} \sum_{k} \sum_{d} \sum_{g} P \operatorname{Cost}_{b, k, d, g} *\left(\sum_{j} R_{j, b, k, d, g}\right)
\end{aligned}
$$

However, if there is available data to integrate the cost reduction by learning experience depending on accumulated production; it means if the learning factor $\beta$ is known for each production technology ( $x$ represents the count of produced products); previous equation should be replaced by:

$$
\text { ProdCost }=\sum_{n} \sum_{j} \sum_{c} \sum_{f} C_{0, n, j, c, f} * \sum_{x=1}^{\sum_{i} N_{i, n, j, c, f}} x^{\beta_{c}}+\sum_{b} \sum_{k} \sum_{d} \sum_{g} C_{0, b, k, d, g} * \sum_{x=1}^{\sum_{j} R_{b, j, k, d, g}} x^{\beta_{d}}
$$

The total pollution cost is composed by the amounts of atmospheric pollutant emissions, residual water generation and solid waste production: 


$$
\begin{aligned}
\text { TPolCostAtm }= & \sum_{x} \text { PolCostA }_{x} \\
& *\left[\sum_{n} \sum_{c} \sum_{f} \phi_{x, n, c, f} *\left(\sum_{j} \sum_{i} N_{i, n, j, c, f}\right)+\sum_{b} \sum_{d} \sum_{g} \phi_{x, b, d, g} *\left(\sum_{k} \sum_{j} R_{j, b, k, d, g}\right)\right] \\
\text { TPolCostWat }= & \sum_{y} \operatorname{PolCost}_{y} \\
& *\left[\sum_{n} \sum_{c} \sum_{f} \psi_{y, n, c, f} *\left(\sum_{j} \sum_{i} N_{i, n, j, c, f}\right)+\sum_{b} \sum_{d} \sum_{g} \psi_{y, b, d, g} *\left(\sum_{k} \sum_{j} R_{j, b, k, d, g}\right)\right] \\
\text { TPolCostSolid }= & \left.\left.\sum_{z} \operatorname{PolCostS_{z}}\right)\right] \\
& *\left[\sum_{n} \sum_{c} \sum_{f} \omega_{z, n, c, f} *\left(\sum_{j} \sum_{i} N_{i, n, j, c, f}\right)+\sum_{b} \sum_{d} \sum_{g} \omega_{z, b, d, g} *\left(\sum_{k} \sum_{j} R_{j, b, k, d, g}\right)\right]
\end{aligned}
$$

Finally, the Initial investment is composed by the investment required to install the pretreatment and the principal production plants:

$$
\text { Initial Investment }=\sum_{j} \sum_{c} \sum_{f} I n v_{j, c, f} * P P_{j, c, f}+\sum_{k} \sum_{d} \sum_{g} I n v_{k, d, g} * W_{k, d, g}
$$

Minimization of the governmental expenditures

Eq. (21)

$$
\begin{aligned}
\operatorname{Min}\left\{\operatorname{IncP}_{j}+\operatorname{IncW}_{k}+\sum_{b} \operatorname{TaxRed}_{b} *\left(\sum_{j} \sum_{m} T_{j, b, m}\right)+\operatorname{GovInvE}\right. \\
\left.+\sum_{a} \operatorname{TaxRed}_{a} *\left(\sum_{k} \sum_{l} P_{k, a, l}\right)+\sum_{c} \operatorname{InvTechP}_{c}+\sum_{d} \operatorname{InvTechW}_{d}\right\}
\end{aligned}
$$

Maximization of the use of nonfood crops as raw materials

Eq. (22)

$$
\operatorname{Max}\left\langle\sum_{n_{N F C}}\left(\sum_{i} \sum_{j} \sum_{c} \sum_{f} N_{i, n, j, c, f}\right)\right\rangle
$$

Maximization of the demand satisfaction with biobased products

Eq. (23)

$$
\operatorname{Max}\left\{\sum_{j} \sum_{b} \sum_{m} T_{j, b, m}+\sum_{k} \sum_{a} \sum_{l} P_{k, a, l}\right\}
$$

Maximization of the raw materials used obtained from land with certification related to sustainable raw material production

Eq. (24)

$$
\operatorname{Max}\left\{\sum_{i} \sum_{n}\left[\text { Certificate }_{i, n} * \sum_{j} \sum_{c} \sum_{f}\left(N_{i, n, j, c, f}\right)\right]\right\}
$$

Minimization of the total amount of raw materials used

Eq. (25) 


$$
\operatorname{Min}\left\{\sum_{n} \sum_{i} \sum_{j} \sum_{c} \sum_{f} N_{i, n, j, c, f}\right\}
$$

Minimization of the total amount of water used for raw materials and biorefinery production Eq. (26)

$$
\begin{gathered}
\operatorname{Min}\left\{\sum_{n} \text { WRawMat }_{n} *\left[\sum_{i} \sum_{j} \sum_{c} \sum_{f} N_{i, n, j, c, f}\right]+\sum_{n} \sum_{c} \sum_{f} \text { WPret }_{n, c, f} *\left[\sum_{i} \sum_{j} N_{i, n, j, c, f}\right]\right. \\
\left.+\sum_{b} \sum_{d} \sum_{g} \text { WPlant }_{b, d, g} *\left[\sum_{j} \sum_{k} R_{j, b, k, d, g}\right]\right\}
\end{gathered}
$$

Minimization of the total amount of water used for the biorefinery production

Eq. (27)

$$
\begin{array}{r}
\operatorname{Min}\left\{\sum_{n} \sum_{c} \sum_{f} \text { WPret }_{n, c, f} *\left[\sum_{i} \sum_{j} N_{i, n, j, c, f}\right]\right. \\
\left.+\sum_{b} \sum_{d} \sum_{g} \text { WPlant }_{b, d, g} *\left[\sum_{j} \sum_{k} R_{j, b, k, d, g}\right]\right\}
\end{array}
$$

Minimization of the locations selected in lands with conflicts

Eq. (28)

$$
\begin{aligned}
& \operatorname{Min}\left\{\sum_{i} \text { ConfLand }_{i} *\left(\frac{\sum_{n} \sum_{j} \sum_{c} \sum_{f} N_{i, n, j, c, f}}{\sum_{n} \sum_{j} \sum_{c} \sum_{f} N_{i, n, j, c, f}+\varepsilon}\right)+\sum_{j} \text { ConfLand }_{j} * \sum_{c} \sum_{f}\left(P P_{j, c, f}\right)\right. \\
& \left.+\sum_{k} \operatorname{ConfLand}_{k} * \sum_{d} \sum_{g}\left(W_{k, d, g}\right)\right\}
\end{aligned}
$$

Minimization of the average value of Gini index for land possession related to the locations selected

Eq. (29)

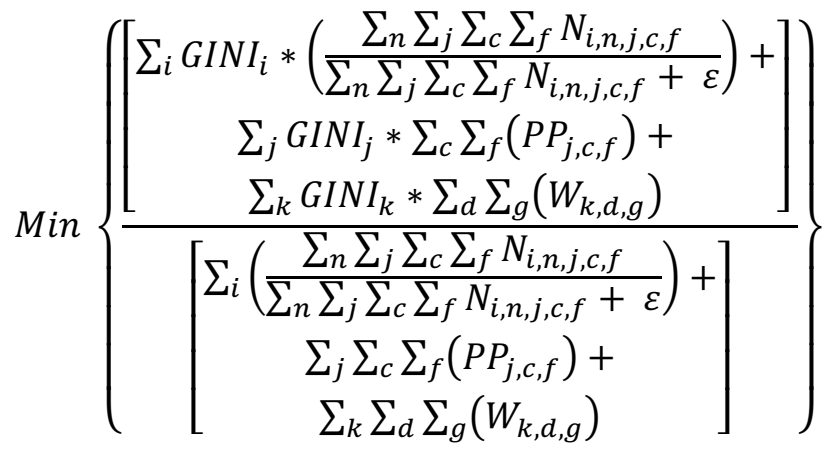

Maximization of the amount of direct and indirect work generated by the biorefinery for the raw material production and at pretreatment and principal production plants

Eq. (30) 


$$
\begin{aligned}
\operatorname{Max}\left\{\sum_{i} \sum_{n}\right. & {\left[\operatorname{DWork}_{R M, i, n}+\operatorname{IWork}_{R M, i, n}\right] *\left[\sum_{j} \sum_{c} \sum_{f}\left(N_{i, n, j, c, f}\right)\right] } \\
& +\sum_{c} \sum_{f}\left[\text { DWork }_{\text {Pret }, j, c}+\text { IWork }_{\text {Pret }, c, f}\right] *\left[\sum_{j} P P_{j, c, f}\right] \\
& \left.+\sum_{d} \sum_{g}\left[\text { DWork }_{\text {Princ }, d, g}+\text { IWork }_{\text {Princ }, d, g}\right] *\left[\sum_{k} W_{k, d, g}\right]\right\}
\end{aligned}
$$

Minimization of the total gas emissions amount related to the consumption of biobased products Eq. (31)

$$
\operatorname{Min}\left[\sum_{a} \operatorname{GasEm}_{\rho, a} * \sum_{k} \sum_{l} P_{k, a, l}+\sum_{b} \operatorname{GasEm}_{\rho, b} * \sum_{j} \sum_{m} T_{j, b, m}\right]
$$

Maximization of the total amount of water recycled used in the biorefinery

Eq. (32)

$$
\begin{aligned}
\operatorname{Max}\left\{\sum_{n} \sum_{c} \sum_{f} R W a t P P_{n, c, f} *\right. & \left.\sum_{i} \sum_{j} N_{i, n, j, c, f}\right] \\
& \left.+\sum_{b} \sum_{d} \sum_{g} R W a t W_{b, d, g} *\left[\sum_{j} \sum_{k} R_{j, b, k, d, g}\right]\right\}
\end{aligned}
$$

Minimization of the water degradation due to raw materials type and location production (water pollution type: $\tau$ )

Eq. (33)

$$
\operatorname{Min} \sum_{n} \sum_{i} \vartheta_{\tau, i, n} *\left(\sum_{j} \sum_{c} \sum_{f} N_{i, n, j, c, f}\right)
$$

Minimization of the sewage water generated

Eq. (34)

$$
\operatorname{Min}\left[\sum_{n} \sum_{c} \sum_{f} S W_{n, c, f} *\left(\sum_{j} \sum_{i} N_{i, n, j, c, f}\right)+\sum_{b} \sum_{d} \sum_{g} S W_{b, d, g} *\left(\sum_{k} \sum_{j} R_{j, b, k, d, g}\right)\right]
$$

Minimization of hazardous and non-hazardous waste generation

Eq. (35)

$$
\operatorname{Min}\left[\sum_{n} \sum_{c} \sum_{f} \omega_{z, n, c, f} *\left(\sum_{j} \sum_{i} N_{i, n, j, c, f}\right)+\sum_{b} \sum_{d} \sum_{g} \omega_{z, b, d, g} *\left(\sum_{k} \sum_{j} R_{j, b, k, d, g}\right)\right]
$$

Maximize the amount of waste recovered or valorized

Eq. (36)

$$
\begin{aligned}
\operatorname{Max} \sum_{z}\left[\sum_{c} \sum_{f} \operatorname{WsRec}_{z, c, f} * \sum_{n}\left\{\omega_{z, n, c, f} *\left(\sum_{j} \sum_{i} N_{i, n, j, c, f}\right)\right\}\right. \\
\left.+\sum_{d} \sum_{g} W_{s R e c} W_{z, d, g} * \sum_{b}\left\{\omega_{z, b, d, g} *\left(\sum_{k} \sum_{j} R_{j, b, k, d, g}\right)\right\}\right]
\end{aligned}
$$

Minimization of the soil deterioration due raw materials cultivation

Eq. (37) 


$$
\operatorname{Min} \sum_{n} \varepsilon_{\zeta, n} *\left(\sum_{i} \sum_{j} \sum_{c} \sum_{f} N_{i, n, j, c, f}\right)
$$

Minimization of the total amount of equivalent $\mathrm{CO}_{2}$ generated at the whole biorefinery supply chain

Eq. (38)

$$
\begin{aligned}
& \operatorname{Min}\left\{\sum_{i} \sum_{n} \mathrm{CO}_{2_{i, n}} *\left(\sum_{j} \sum_{c} \sum_{f} N_{i, n, j, c, f}\right)+\sum_{n} \sum_{c} \sum_{f} \mathrm{CO}_{2_{n, c, f}} *\left(\sum_{j} \sum_{i} N_{i, n, j, c, f}\right)\right. \\
& +\sum_{b} \sum_{d} \sum_{g} \mathrm{CO}_{2_{b, d, g}} *\left(\sum_{k} \sum_{j} R_{j, b, k, d, g}\right)+\sum_{a} \mathrm{CO}_{2} *\left(\sum_{k} \sum_{l} P_{k, a, l}\right) \\
& +\sum_{b} \mathrm{CO}_{2_{b}} *\left(\sum_{j} \sum_{m} T_{j, b, m}\right)+\sum_{i} \sum_{n} \sum_{j} \mathrm{CO}_{2 i, n, j} *\left(\sum_{c} \sum_{f} N_{i, n, j, c, f}\right) \\
& +\sum_{j} \sum_{b} \sum_{k} \mathrm{CO}_{2_{j, b, k}} *\left(\sum_{d} \sum_{g} R_{j, b, k, d, g}\right)+\sum_{k} \sum_{a} \sum_{l} \mathrm{CO}_{2, a, l} * P_{k, a, l} \\
& \left.+\sum_{j} \sum_{b} \sum_{m} \mathrm{CO}_{2, b, m} * T_{j, b, m}\right\}
\end{aligned}
$$

Minimization of the amount of land used for the raw materials production

Eq. (39)

$$
\operatorname{Min}\left\{\sum_{n} \sum_{i} \frac{\sum_{j} \sum_{c} \sum_{f} N_{i, n, j, c, f}}{\text { RMYield }_{i, n}}\right\}
$$

Minimization of the annual consumption of fuels in the entire biorefinery supply chain

Eq. (40)

Where

$$
\operatorname{Min} \frac{[(a)+(b)+(c)]}{[(d)+(e)+(f)]}
$$

$$
\begin{gathered}
(a)=\sum_{i} \sum_{n} N R F_{i, n} *\left[\sum_{j} \sum_{c} \sum_{f} N_{i, n, j, c, f}\right] \\
(b)=\sum_{n} \sum_{c} \sum_{f} \operatorname{NRFPret}_{n, c, f} *\left[\sum_{j} \sum_{i} N_{i, n, j, c, f}\right]+\sum_{b} \sum_{d} \sum_{g} \operatorname{NRFPrinc}_{b, d, g} *\left[\sum_{k} \sum_{j} R_{j, b, k, d, g}\right] \\
(c)=\sum_{n} \sum_{i} \sum_{j} \operatorname{NRFLog}_{n, i, j} *\left[\sum_{c} \sum_{f} N_{i, n, j, c, f}\right]+\sum_{b} \sum_{j} \sum_{k} N R F \log _{b, j, k} *\left[\sum_{d} \sum_{g} R_{j, b, k, d, g}\right] \\
+\sum_{a} \sum_{k} \sum_{l}\left(N R F \log _{a, k, l} * P_{k, a, l}\right)+\sum_{b} \sum_{j} \sum_{m}\left(N R F \log _{b, j, m} * T_{j, b, m}\right) \\
(d)=\sum_{i} \sum_{n}\left[R F_{i, n}+N R F_{i, n}\right] *\left[\sum_{j} \sum_{c} \sum_{f} N_{i, n, j, c, f}\right]
\end{gathered}
$$




$$
\begin{aligned}
(e)=\sum_{n} \sum_{c} \sum_{f}\left[\operatorname{RFPret}_{n, c, f}+\operatorname{NRFPret}_{n, c, f}\right] *\left[\sum_{j} \sum_{i} N_{i, n, j, c, f}\right] \\
+\sum_{b} \sum_{d} \sum_{g}\left[\operatorname{RFPrinc}_{b, d, g}+\operatorname{NRFPrinc}_{b, d, g}\right] *\left[\sum_{k} \sum_{j} R_{j, b, k, d, g}\right] \\
(f)=\sum_{n} \sum_{i} \sum_{j}\left[\operatorname{NRFLog}_{n, i, j}+\operatorname{RFLog}_{n, i, j}\right] *\left[\sum_{c} \sum_{f} N_{i, n, j, c, f}\right] \\
+\sum_{b} \sum_{j} \sum_{k}\left[\operatorname{NRFLog}_{b, j, k}+R F \log _{b, j, k}\right] *\left[\sum_{d} \sum_{g} R_{j, b, k, d, g}\right] \\
+\sum_{a} \sum_{k} \sum_{l}\left[\operatorname{NRFLog}_{a, k, l}+R F \log _{a, k, l}\right] * P_{k, a, l} \\
+\sum_{b} \sum_{j} \sum_{m}\left[\operatorname{NRFLog}_{b, j, m}+R F \log _{b, j, m}\right] * T_{j, b, m}
\end{aligned}
$$

Minimization of the energy entered to the energy balance in the biorefinery

Eq. (41)

$$
\operatorname{Min}\left\{\begin{array}{c}
\sum_{n}\left[\left(\sum_{i} \sum_{j} \sum_{c} \sum_{f} N_{i, n, j, c, f}\right) * \theta_{n}\right]+\sum_{i} \sum_{j}\left[\left(\sum_{n} \sum_{c} \sum_{f} N_{i, n, j, c, f}\right) * \delta * \text { Dist }_{i, j}\right]+ \\
\sum_{n} \sum_{c} \sum_{f}\left[\left(\sum_{i} \sum_{j} N_{i, n, j, c, f}\right) * \beta_{n, c, f}\right]+\sum_{j} \sum_{k}\left[\left(\sum_{b} \sum_{d} \sum_{g} R_{j, b, k, d, g}\right) * \delta * \text { Dist }_{j, k}\right]+ \\
\sum_{b} \sum_{d} \sum_{g}\left[\left(\sum_{j} \sum_{k} R_{j, b, k, d, g}\right) * \beta_{b, d, g}\right]+\sum_{k} \sum_{l}\left[\left(\sum_{a} P_{k, a, l}\right) * \delta * \operatorname{Dist}_{k, l}\right]+ \\
\sum_{j} \sum_{m}\left[\left(\sum_{b} T_{j, b, m}\right) * \delta * \operatorname{Dist}_{j, m}\right]-\sum_{b}\left[\left(\sum_{j} \sum_{m} T_{j, b, m}\right) * \theta_{b}\right]- \\
\sum_{b}\left[\left(\sum_{j} \sum_{c} \operatorname{Rec}_{b, j, c}\right) * \theta_{b}\right]-\sum_{a}\left[\left(\sum_{k} \sum_{l} P_{k, a, l}\right) * \theta_{a}\right]-\sum_{a}\left[\left(\sum_{k} \sum_{d} \operatorname{Reu}_{a, k, d}\right) * \theta_{a}\right]
\end{array}\right\}
$$

Maximization of the raw material recycled used in the biorefinery

Eq. (42)

$$
\operatorname{Max} \sum_{i} \sum_{n} \operatorname{RecRM}_{i, n} *\left(\sum_{j} \sum_{c} \sum_{f} N_{i, n, j, c, f}\right)
$$

\subsubsection{Supply chain design: Model components}

SC model related to the strategic decision-making level is characterized by a set of decision variables and parameters graphically described in figure 5. This SC includes the suppliers, the pretreatment plants, the principal production plants and the clients for marketable products obtained in pretreatment (for example refined oils) and principal production plants. 


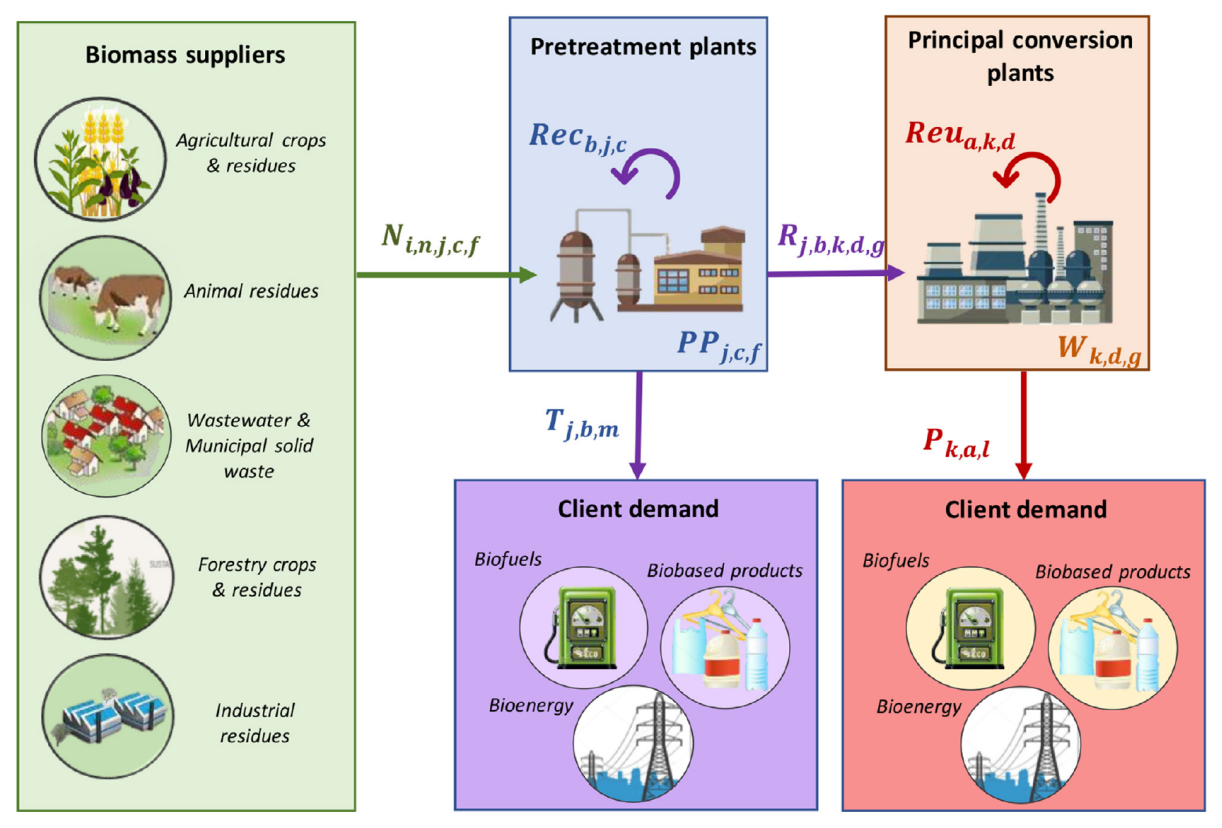

Figure 5. SC configuration related to the strategical decision-making level for Phase III Biorefinery

The SC restrictions correspond to mass balances, raw material availability, production capacity limits, selection of capacity and production technology, demand limitations and variable restrictions.

Constraints represented by Eq. (1) and Eq. (2) are an application of the law of conservation of mass to the analysis of physical systems. Therefore, there exists a mass balance for each pretreatment plant, mathematically expressed by Eq. (1), and for the main production plants, represented by Eq. (2). These balances have to be made by differentiating the type of product obtained, as a consequence of the different transformation rates, which depend on income materials type and the applied transformation technology.

Raw materials to be consumed in biorefineries are limited by its availability at each supplier location as described by Eq. (3).

Eq. (4) and Eq. (5) represent the constraints related to production capacity at pretreatment plants and principal plants respectively. This means that the amount of incoming materials is limited by the processing capacity. And the installations can only receive materials if the plant has been installed with a specific technology and capacity.

Also, the selections of production capacity and technology for each location have to be integrated. Because only one production capacity and one transformation technology can be selected for each plant location, Eq. (6) and Eq. (7) represents the constraints for the pretreatment plants and the main production plants technologies, respectively.

Decisions related to strategical decision-level SC does not include inventory decision. Therefore, it is not possible to transport more products than the demanded amount at each final selling point, as presented in equations (8) and (9). However, in models including tactical and operational decisions, inventory management is a key decision.

Finally, some variable restrictions have been included because the products to be sold only can be produced in a plant if this plant is already installed. Therefore, restrictions Eq. (10) and Eq. (11) are required for pretreatment plants. Also, Eq. (12) constraint is required to principal plants. 
These restrictions and the mass balance contribute to limit the values of $\operatorname{Rec}_{b, j, c}$ and $\operatorname{Reu}_{a, k, d}$, which could only be different from zero if the corresponding production plants are installed.

\subsubsection{Sustainability model components}

In order to determine the quantitative measures that can be represented as mathematical expressions for the optimization model, the framework developed by Bautista et al., (2016) and the Global Reporting Initiative, (2016) were analyzed from the point of view of the strategical SC decision-making level. It means, considering if each indicator or measure can be estimated before the implementation of the biorefinery. The results of this analysis and modeling are: (i) five decision variables description; $\operatorname{Inc} P_{j}$ : the amount of government incentives for setting up a pretreatment plant at location $j ; I_{n} W_{k}$ : the amount of government incentives for setting up a production plant at location $k ; \operatorname{InvTech} P_{c}$ : Amount invested by the government in technology $c$; InvTech $W_{d}$ : Amount invested by the government in technology $d ;$ GovInvE: the governmental investment in electricity infrastructure;; (ii) 97 parameters definition; and (iii) six constraints inclusion, and (iv) twenty-four mathematical expressions defined as objective functions (Eq. (19) to Eq. (42)), which are presented below. The sustainability measures that are not presented could not yet be translated to mathematical expressions for the design of the Phase III Biorefinery SSC.

The constraint related to the maximum budgetary amount of governmental incentives for biorefinery plants installation is represented by Eq. (13). The maximum amount of governmental incentives by pretreatment plant installed is characterized by Eq. (14); it can be a percentage of the investment required for the pretreatment plant or a maximal cap established by each facility. Likewise, the constraint related to the maximum amount of governmental incentives by principal production plant installed is Eq. (15). The maximum budgetary amount of governmental incentives for production technologies development is described by Eq. (16).

The update of bioelectricity demand depending on governmental investments in electric infrastructure is represented by Eq. (17).

Finally, the consumption of water in the biorefinery is limited by the availability of water, as characterize Eq. (18).

Table 2 presents the relation between each objective function and constraint with the sustainability criteria and indicators presented by Bautista et al., (2016) and the Global Reporting Initiative, (2016). It can be noted that the environmental dimension is the most represented in the developed model.

Table 2. Relations between the equations and the sustainability frameworks

\begin{tabular}{|lccc|}
\hline Equation & Indicator (TBL+) & Indicator (GRI) & $\begin{array}{c}\text { Sustainability } \\
\text { dimension }\end{array}$ \\
\hline Eq. (13) & 83 & & Economic \\
\hline Eq. (14) & $84,86,43,104$ & 302.5 & Economic \\
\hline Eq. (15) & 43,48 & & Political \\
\hline Eq. (16) & 45 & & Political \\
\hline Eq. (17) & 46 & Political \\
\hline Eq. (18) & 50 & & Political \\
\hline Eq. (19) & 102 & Technological \\
\hline Eq. (20) & 103 & & Technological \\
\hline Eq. (21) & 103 & 411.1 & Technological \\
\hline Eq. (22) & $1,2,3,25$ & Social \\
\hline Eq. (23) & 7,22 & 413.1 & Social \\
\hline Eq. (24) & $18,35,36$ & 305.1 & Social \\
\hline Eq. (25) & $107,108,109,110,111,112$ & Environmental \\
\hline Eq. (26) & 117 & 303.3 & Environmental \\
\hline Eq. (27) & $119,120,121,122,124,125$ & $306.1,306.2$ & Environmental \\
\hline
\end{tabular}




\begin{tabular}{|cccc|}
\hline Eq. (28) & 123 & 306.5 & Environmental \\
\hline Eq. (29) & 126,129 & 306.4 & Environmental \\
\hline Eq. (30) & 128 & & Environmental \\
\hline Eq. (31) & $130,131,132,133,134,137$ & 303.1 & Environmental \\
\hline Eq. (32) & $140,141,143$ & $305.1,305.2,305.3,305.4$ & Environmental \\
\hline Eq. (33) & 146 & & Environmental \\
\hline Eq. (34) & 155,156 & $301.1,302.1,302.2,302.3$ & Environmental \\
\hline Eq. (35) & 157 & $302.1,302.2,302.3$ & Environmental \\
\hline Eq. (36) & & 301.2 & Environmental \\
\hline Eq. (37) & 43 & & Political \\
\hline Eq. (38) & 48 & & Political \\
\hline Eq. (39) & 11 & & Social \\
\hline Eq. (40) & 113 & & Environmental \\
\hline
\end{tabular}

\subsection{Solution approach}

The model developed applying the strategy described in the section 3.1 involves twenty-four objective functions. This type of problems are known as Multi-Objective Optimization Problems (MOOP) (Narzisi et al., 2006). The objectives in MOOP are often in conflict with each other. Thus, the improvement of one objective may lead to the deterioration of another (Zhou et al., 2011). One solution optimal is one that is non-dominated by any other in the analysis space (Suárez Palacios et al., 2011). Thus, there is no single optimum solution. Instead there is a set of solutions which are all optimal, called the Optimal Pareto Front (Narzisi et al., 2006).

There are different ways to find the Pareto Front (PF) (Hillier and Lieberman, 2001). However, evolutionary algorithms (EAs) has gained attention, because they are able to approximate the whole PF of a MOOP in a single run (Huband et al., 2006; Zhou et al., 2011). It is due to their population-based nature that gives them the ability to handle complex problems, involving features such as discontinuities, multimodality, disjoint feasible spaces and noisy function evaluations (Fonseca and Fleming, 1995). Several EAs can be used to solve MOOPs; nevertheless, the most representative algorithm is Non-Dominated Sorting Genetic Algorithm (NSGA-II) (Espinoza Pérez, 2017). This algorithm uses an elitist principle, i.e. it incorporates a mechanism for preserving the dominant solutions through several generations of a genetic algorithm; in addition to an explicit diversity preserving mechanism; and emphasis in nondominated solutions.

Particular attention should be paid to the amount of objective functions to compare by optimization. Because, in multiobjective problems graphical representation of the optimization results has a great importance in the analysis and decision making process (Blasco et al., 2017). In fact, depending on the number of objective functions to be optimized and the type of graphic to be performed there will be a number of possible combinations. For example, for 3 objective functions there are 3 combinations for 2D graphs and only one combination for a 3D graph. However, for 20 objective functions, there are 190 combinations for 2D graphs and 1140 combination for a 3D graph. It might be concluded that if the total amount of objective functions to optimize are more than five, it is recommendable to use 2D graphics to represent the solutions. These 2D graphics would help stakeholders to perform a ranking among the objectives functions to select an optimal biorefinery SC.

\section{Case study description and implementation}

In order to evaluate the performance of the developed model, a case study of the development of industrial biorefineries in Colombia is developed. Indeed, since the early 2000 Colombian government has decided to invest in a biodiesel sector. Today more than 450000 tons of biodiesel are produced from palm oil under traditional conditions (Fedebiocombustibles, 2017). So the aim 
of this study is to explore conditions for a more sustainable and higher added value sector through a Phase III Biorefinery SSC.

\subsection{Colombian case study}

More precisely this country was selected because: (1) there are Phase II Biorefineries currently in production; (2) there is a market regulation for biobased products, such as biodiesel (Costa et al., 2017); (3) the biofuel industry is mainly funded by government subsidies; (4) the principal raw material used to produce biodiesel is the food crop palm oil, producing conflicts for soil use and crops use (Costa et al., 2017), (5) enterprises consider in very rare cases the possibility of selling products with high added value (Bueno et al., 2015).

The idea pursued in the case study is to diversify the raw materials to be used, comparing palm and jatropha oil (Hernández Castiblanco and Amórtegui Gómez, 2015). Also, diversifying the final products to be obtained (Bueno et al., 2014), assessing biodiesel, glycerol and aliphatic polyester production. In addition, different production technologies are assessed (Basto Aluja, 2016), considering the industrialized alkaline transesterification, but also emerging technologies such as the transesterification counter-current and co-current in development for the biodiesel production (J.G.Cadavid et al., 2013; Narváez et al., 2009), and the production technology in development that allows to transform glycerol into bio-based polymer (Bueno et al., 2014). The selected geographical area for the case study is the northern region of Colombia, which represent one of the most important palm crops areas. In this regard, the potential supplier locations and biomass availability considered are presented in table 3 . Likewise, table 4 presents the market potential location and demand for the final and intermediate products. Nine locations are proposed for the installation of production plants and eight locations are proposed for the pretreatment plants in order to refine the oil, as presented in figure 6 .

Table 3. Potential supplier locations and biomass availability in northern Colombia in 2015. Based on Fedepalma, (2016)

\begin{tabular}{|c|c|c|}
\hline Location & $\begin{array}{c}\text { Raw material } \\
\text { type }\end{array}$ & $\begin{array}{c}\text { Estimated } \\
\text { availability } \\
\text { (Hectares/year) }\end{array}$ \\
\hline \multirow{2}{*}{$\begin{array}{c}\text { Bosconia / } \\
\text { Cesar }\end{array}$} & Palm Oil & 68,111 \\
\hline & Jatropha Oil & 0 \\
\hline \multirow{2}{*}{$\begin{array}{c}\text { María La Baja / } \\
\text { Bolívar }\end{array}$} & Palm Oil & 25,899 \\
\hline & Jatropha Oil & 31,425 \\
\hline \multirow{2}{*}{$\begin{array}{l}\text { Barrancabermeja } \\
\text { / Santander }\end{array}$} & Palm Oil & 63,044 \\
\hline & Jatropha Oil & 0 \\
\hline \multirow{2}{*}{$\begin{array}{c}\text { Montería / } \\
\text { Córdoba }\end{array}$} & Palm Oil & 0 \\
\hline & Jatropha Oil & 286,738 \\
\hline \multirow{2}{*}{ Codazzi / César } & Palm Oil & 0 \\
\hline & Jatropha Oil & 137,356 \\
\hline \multirow{2}{*}{$\begin{array}{l}\text { Sincelejo / } \\
\text { Sucre }\end{array}$} & Palm Oil & 0 \\
\hline & Jatropha Oil & 72,856 \\
\hline \multirow{2}{*}{$\begin{array}{c}\text { Santa Marta / } \\
\text { Magdalena }\end{array}$} & Palm Oil & 45,834 \\
\hline & Jatropha Oil & 48,250 \\
\hline \multirow{2}{*}{$\begin{array}{c}\text { Albania / La } \\
\text { Guajira }\end{array}$} & Palm Oil & 0 \\
\hline & Jatropha Oil & 45,756 \\
\hline
\end{tabular}

Table 4. Market potential location and demand in northern Colombia in 2015. Base on Fedebiocombustibles, (2015) and DANE, (2017)

\begin{tabular}{l|c|c|} 
Location & Product demanded & Demand (Ton/year) \\
\hline
\end{tabular}




\begin{tabular}{|c|c|c|c|}
\hline \multirow{2}{*}{ 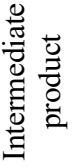 } & Barranquilla (1) & Refined palm oil & 10,072 \\
\hline & Santa Marta (2) & Refined palm oil & 55,758 \\
\hline \multirow{21}{*}{ 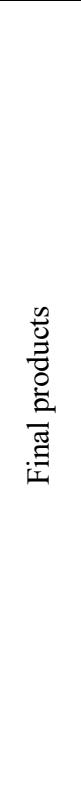 } & \multirow{3}{*}{ Montería (1) } & Biodiesel & 25,206 \\
\hline & & Glycerol & 0 \\
\hline & & Polymer & 0 \\
\hline & \multirow{3}{*}{ Sincelejo (2) } & Biodiesel & 12,554 \\
\hline & & Glycerol & 0 \\
\hline & & Polymer & 0 \\
\hline & \multirow{3}{*}{ Cartagena (3) } & Biodiesel & 30,920 \\
\hline & & Glycerol & 0 \\
\hline & & Polymer & 1,515 \\
\hline & \multirow{3}{*}{ Barranquilla (4) } & Biodiesel & 36,282 \\
\hline & & Glycerol & 8 \\
\hline & & Polymer & 605 \\
\hline & \multirow{3}{*}{ Santa Marta (5) } & Biodiesel & 18,574 \\
\hline & & Glycerol & 0 \\
\hline & & Polymer & 0 \\
\hline & \multirow{3}{*}{ Valledupar (6) } & Biodiesel & 15,169 \\
\hline & & Glycerol & 0 \\
\hline & & Polymer & 0 \\
\hline & \multirow{3}{*}{ Bucaramanga (7) } & Biodiesel & 30,388 \\
\hline & & Glycerol & 0 \\
\hline & & Polymer & 4 \\
\hline
\end{tabular}

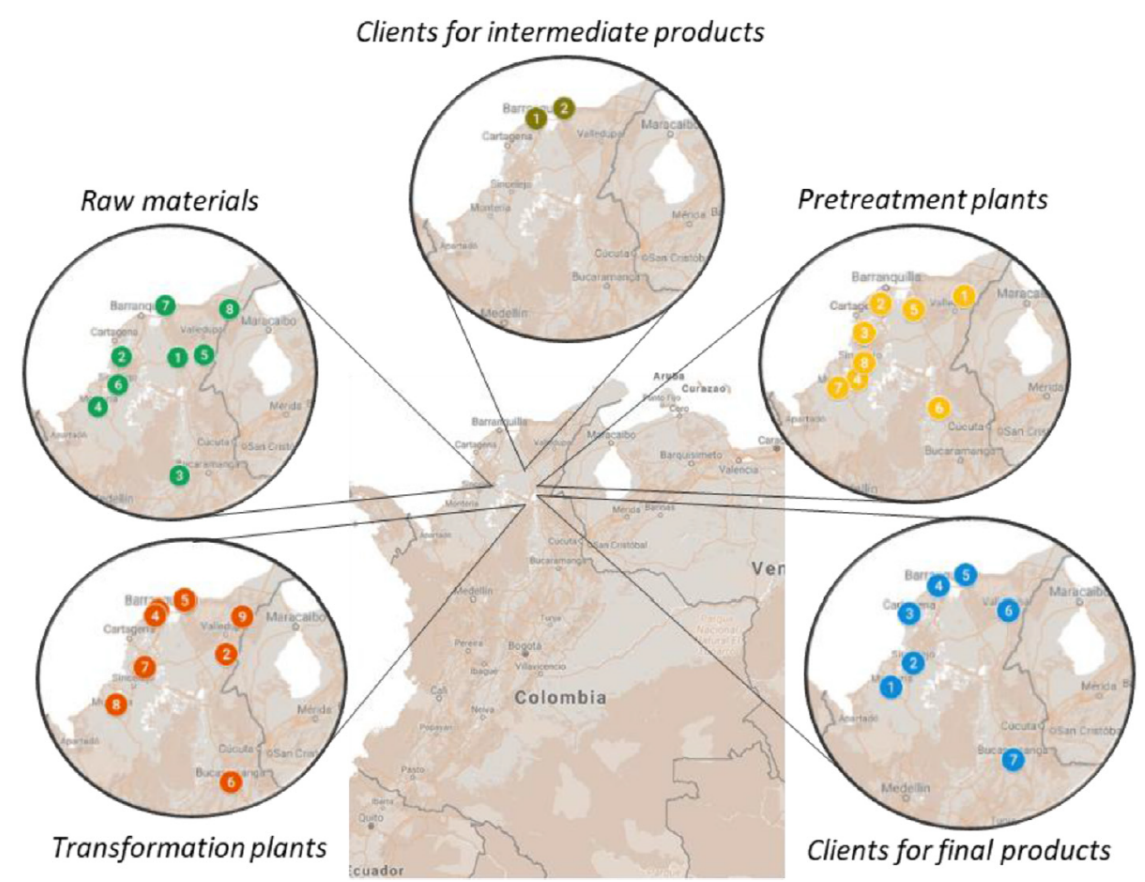

Figure 6. Locations for the Phase III Biorefinery SSC optimization model application

\subsection{Results and discussion}

The proposed model applied to the Colombian case study is integrated by 3,459 decision variables, including 186 binary variables. In addition, there are 43 equality constraints and 287 
inequality constraints. There are twenty-two objective functions; considering Nitrogen, Phosphorus and Phosphates representing the general objective function Eq. (37). Eq. (32) could not be represented due, because production technologies assessing water recycle were not included. Likewise, Eq. (36) could not be represented, because production technologies assessing waste recovering were not included. Besides, there is not raw material recycled defined in the case study to apply Eq. (42) and there are no data available to apply constraints Eq. (17) and (18).

Particular attention should be paid to the amount of objective functions to compare by optimization. Because, in multiobjective problems graphical representation of the optimization results has a great importance in the analysis and decision-making process (Blasco et al., 2017). In fact, depending on the number of objective functions to be optimized and the type of graphic to be performed there will be a number of possible combinations. To explain better explain, Table 5 shows the quantity of graphics that will be generated depending on the total number of objective functions and the graphic type.

Table 5. Objective combination

\begin{tabular}{|c|c|c|}
\hline \multirow{2}{*}{$\begin{array}{l}\text { Total objective } \\
\text { function amount }\end{array}$} & \multicolumn{2}{|c|}{ Graphic type } \\
\hline & 2D: Two functions & 3D: Three functions \\
\hline 3 & 3 & 1 \\
\hline 5 & 10 & 10 \\
\hline 10 & 45 & 120 \\
\hline 20 & 190 & 1,140 \\
\hline 30 & 435 & 4,060 \\
\hline
\end{tabular}

Thus, it might be concluded that if the total amount of objective functions to optimize is more than five, it is recommendable to use 2D graphics to represent the solutions. Consequently, there are 253 possible combinations for all the defined objective functions in the Colombian case study. In this way, the multiobjective model was implemented in MatLab ${ }^{\circledR}$ to compare objective functions by pairs. The case study was solved using the algorithm NSGA-II in an Intel Core i7$6700 \mathrm{HQ}, 2.6 \mathrm{GHz}$ computer in $16 \mathrm{~GB}$ RAM.

\subsubsection{Pareto fronts}

In order to develop a first analysis, the objective function that maximizes the net present value (NPV), was chosen to be compared to the remaining objective functions. Thus, with the purpose of facilitating the interpretation of each chart; Figure 7 shows all the Pareto fronts in the interval [-75; 75] million dollars for NPV. Despite Eq. (15), (18), (20), (26) and (37) should be plotted in the interval $[-4,000 ; 500]$ million dollars for NPV. Since, if all objective functions are graphed in this last interval, most of the pairwise comparison will not be seen clearly to allow its analyses. 


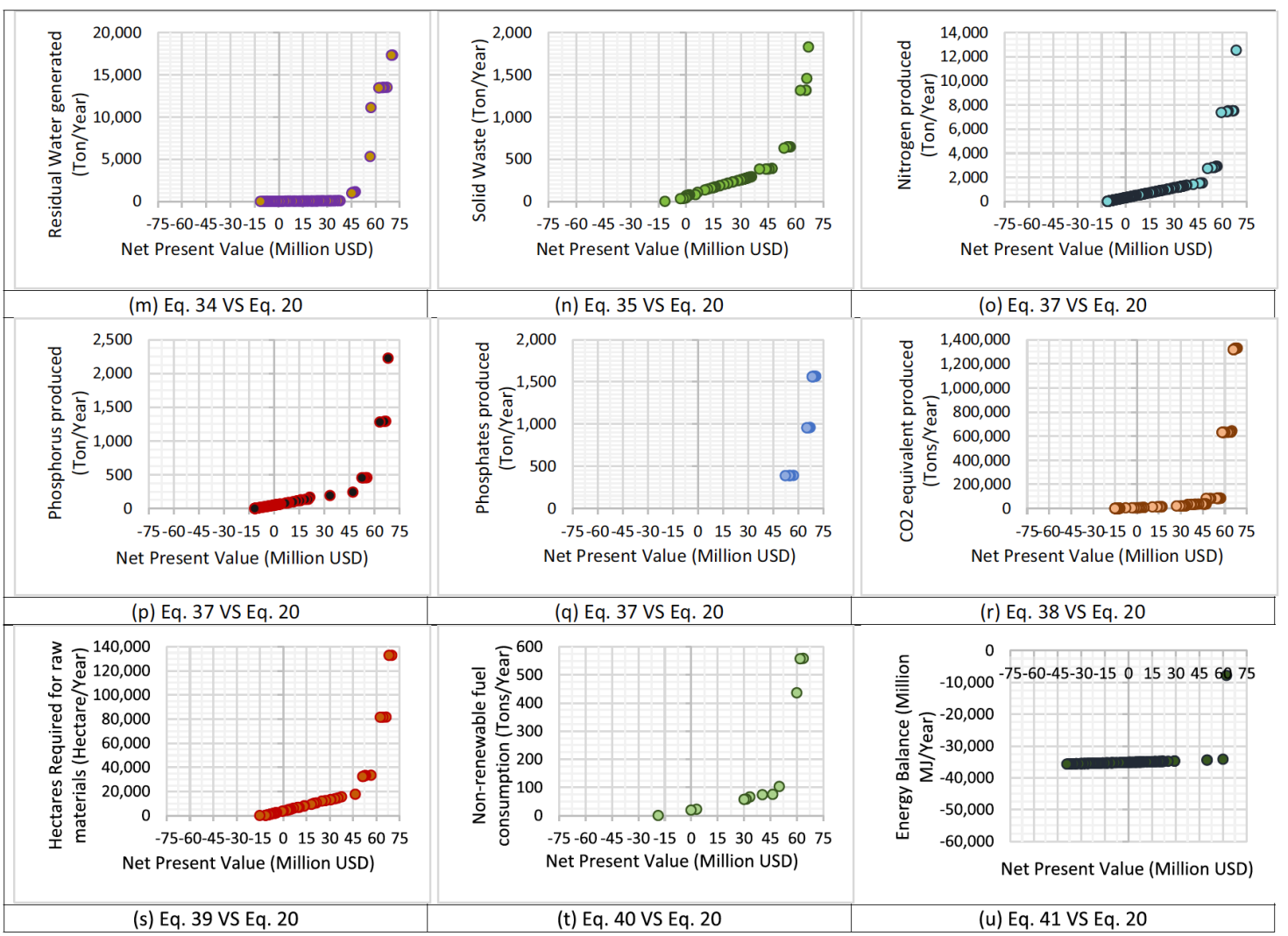

Figure 7. Pareto fronts for the case study

Given the amount of information offered through the developed optimization model, a detailed discussion of the results is not possible to be presented in this paper. Instead, the most interesting results are discussed, highlighting the core contributions of this work below:

Figure (a). It shows that the maximum value of NPV is reached at $93 \%$ of the installed capacity is used.

Figure (b). Governmental expenses increase when the NPV rises, due in $99 \%$ to the biodiesel tax reduction, reaching 22,000,000 USD/year.

Figure (c). It shows that diversification of raw materials with non-food crops can be economically feasible in the presented case study. However, in order to maximize the NPV lower amounts of non-food crops should be used in the production process (To maximize the NPV only around 200,000 tons of non-food crops are used, however, there are around 850,000 tons available). This is mainly because transformation rates are lower in the case of jatropha at pretreatment and principal production plants. It shows the impact of production technologies in the phase III biorefinery development.

Figure (d). In order to obtain a better economic performance the satisfactions of the total final product demand should only reach $85 \%$. However, the high-value products demand (aliphatic polymer) is completely satisfied. It shows that the high-value products contribute to reach higher economic performance.

Figure (e). The number of selected certified hectares decreases when economic results improve, because only some of the palm suppliers are certified in RSPO. Despite the certified land availability is enough to produce the oil required for the total biodiesel demand satisfaction. Thus, it can be concluded that the transportation cost is an important factor in economic terms, entailing the importance of tactical and operational decisions.

Figures (f) and (s). Around 250,000 tons of raw materials are required to maximize the NPV. This represents the $16 \%$ of the total potential availability of jatropha and palm crude oil (around 850,000 t/year and 700,000 t/year, respectively). Thus, the potential raw material availability will satisfy the demand from other regions of Colombia. 
Figures (g), (h), (m), (n) and (t). In the same manner, the use of (h) process water, (t) fuel and $(\mathrm{g})$ total water, also will increase when positive economic results are obtained. At the same time, (m) wastewater and (n) solid waste production increases. Because these flows are linked to the production amounts.

Figures (i) and (j). When the hectare requirement rises due to higher values of production to maximize the NPV, areas with high dispersion value will be selected, as shows figure (i). Similarly, if more plants are installed and more suppliers are selected to obtain good economic performance, areas with higher GINI index will be selected as shown by figure (j), reaching an average GINI 0.75 (related to the degree of inequality degree of wealth in a geographical region). However, despite the fact that it is a high value for the index, it must to be considered the special local conditions in the Colombian case, where the average GINI index for the country is 0.734 .

Figure (k). The number of workers decreases when NPV increases, because production plants will be installed only if they allow the stakeholders to obtain better economic performance. Also, the raw material will be the amount required to satisfy the demand that maximizes the economic results. Influencing the number of workers required at cultivation stage.

Figures (l) and (r). To obtain additional incomes, intermediate and final products must to be sold. Consequently, gas emissions, as $\mathrm{CH}_{4}$ and $\mathrm{N}_{2} \mathrm{O}$, increase when NPV rises, due to biobased products use, principally biodiesel (1). Likewise, $\mathrm{CO}_{2}$-equivalent emissions will increase inasmuch as the economical results improve (r). In this analyze, negative values for $\mathrm{CO}_{2}$-equivalent emissions will be caused by $\mathrm{CO}_{2}$ capture at raw material cultivation stage.

Figures (o), (p) and (q). Water pollution generated by phosphorus, phosphates and nitrates increase exponentially due to the higher raw material requirements (f), increasing at the same time the number of required hectares (s).

Figure (u). The comparison between the energy balance and the NPV shows that the net energy consumed (consumption-generation) increases when the economic results improve. This is because energy must to be consumed in logistics, in order to bring products from pretreatment to principal plants when final products will be produced, and energy will be required in transformation processes. In addition, energy must to be consumed in logistics to deliver products to customers.

Summarizing, the Pareto fronts presented in Figure 4 shows that: Phase III biorefineries are economically feasible in North Colombia. It implies, integrating different types of raw materials, even non-food crops, and obtaining several bio-based products. However, if there are evaluated under a pure economic perspective, these SC configurations are not the most optimal. In addition, it is noted that governmental expenses by tax reductions will represent an important value each year, reaching 22 million dollars. Furthermore, emerging technologies present high potential yields to produce biobased products in the case study. Thus, they will be preferred when optimizing economic aspects. However, the risk related to the use of non-industrialized technologies should be integrated in further researches. Finally, since Pareto fronts exist, there are optimal configurations for phase III biorefineries SC that equilibrates objective functions for the case study. Thus, further studies should be made to select the optimal configuration based on decision-maker preferences. In order to determine how important is each sustainability aspect represented by the objective functions.

\subsubsection{Sensitivity analyses}

The sensitivity analysis is an important aspect in model solution because it deals with obtaining additional information about the behavior of the optimum solution when the model undergoes some parameter changes (Taha, 2010). In order to perform the sensitivity analysis there are two types of parameters that can be modified in the developed model. First, parameters related to the integrated model constraints; and second, parameters related to the objective functions.

It should be observed that the potential raw material availability, even considering only the current crude palm oil availability in Colombia, is enough to satisfy the current demand. Therefore, it is 
not a parameter that will affect the optimization results at this stage. Thus, as first attempt, we decide to analyze the behavior of the NPV face to percentage variations in products demand and price. Those values where modified in $-50 \%,-25 \%,+25 \%$ and $+50 \%$. In the sensitivity analysis, it was observed that the NPV is more sensitive to changes in biodiesel price (reaching variations of $-25 \%,-8 \%,+350 \%$ and $+500 \%$ of NPV baseline, respectively) than polymer price, refined palm oil demand, biodiesel demand or polymer demand (whose variations are between $-5 \%$ and $+8 \%$ of NPV baseline).

This behavior is due mainly to the lower demanded amounts for aliphatic polymer and refined palm oil at the local scale. Thus, the integration of international markets in the supply chain configuration could be an interesting development to find more demand sources for high addedvalue products. It was also verified that production plant selection is centralized due required high investment.

\subsection{Model development and solution insights}

The model constraints does no considers a lower bound for the clients demand. Thus, when assessing some minimization objective functions as a sole objective, biorefineries would not be implemented. However, due the multiobjective nature of the sustainability and the solution approach used, the Pareto fronts are constructed and conflicting objective functions are detected, despite the absence of lower bound for the clients demand.

Moreover, the optimization model was developed in a generic form for its implementation in any Phase III Biorefinery SSC case study. Nevertheless, the inclusion of each constraint and objective function related to sustainability depends on the data availability and the case study defined.

In addition, it is noted that the environmental dimension is the most represented, due the amount of objective functions defined. However, several objective functions measure emissions or material flows, i.e. these objective functions measure amounts and not impacts. Hence, in-depth studies should be made related to the objective functions described in this research. In addition, it was observed that there are several objective functions related. Thus, further researches could analyze the relations and the dynamism among the objective functions defining the Phase III biorefinery SSC, in order to better understand the system behavior.

Concerning the model solution, a previous analysis to the solution space is required to optimize simultaneously all the objective functions. To better identify the optimization methodology in order to use the computational resources efficiently.

\section{Conclusions}

This work builds on several research gaps identified in literature. This work: 1) provides an integrated SC design optimization model that incorporates several SC decisions such as supplier selection, raw material type selection, facility location and capacity installation, technology selection, pretreatment plants location and capacity installation, final products market and type selection; 2) explicitly evaluates the sustainability aspects of all the SC activities, especially the indicators used by the GRI Sustainability Reporting Standards and the TBL+; 3) provides a multiobjective decision-making tool that addresses the sustainability aspects allowing the study of their interactions and deriving strategies. This work is a step forward to provide policy makers and investors with a more holistic vision on the implications of a biorefinery project within it territorial context. It also allows exploring trade-off zones of the requirements and priorities of such a multi-stakeholder project. Even though in the current status the optimization model provides a vast amount of information, it should be defined a way to present it that is easily understandable by all the stakeholders.

Although the important step was taken on the definition of Phase III Biorefinery SSC, future work should still be done. Namely, the integration of the dynamic nature of SC, including multiperiod 
aspect, tactical and operational decision-making levels, as well as, the degree of uncertainty involved in internal and external parameters. In addition, at the sustainability analysis level, the impact of different indicators should be analyzed in order to not only consider flows, but also analyze significant impacts.

\section{Acknowledgements}

The authors would like to thank the Chilean scholarship (Becas Chile) from the National Commission for Scientific and Technological Research (CONICYT, Chile) program for their support to Andrea Espinoza in this research work

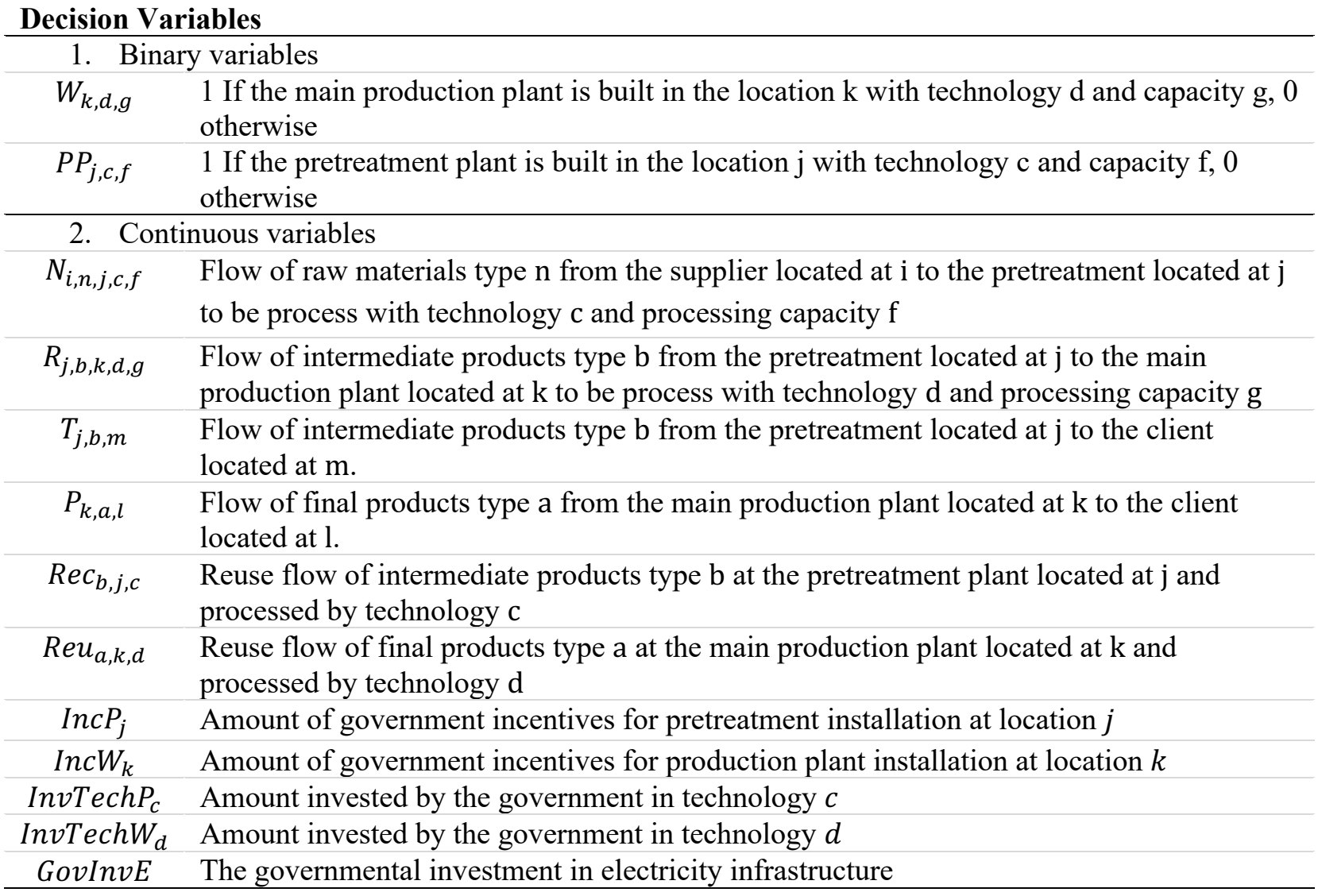

\section{Indices notation}

\begin{tabular}{cl}
\hline \multicolumn{2}{l}{ Indices } \\
\hline$n$ & Feedstock type \\
\hline$i$ & Supplier location \\
\hline$j$ & Pretreatment location \\
$c$ & Production technology at pretreatment \\
\hline$b$ & Intermediate product type \\
\hline$f$ & Transformation capacity at pretreatment \\
$m$ & Intermediate product demand location \\
\hline$k$ & Conversion plants location \\
\hline$d$ & Production technology at conversion plants \\
\hline$a$ & Final product type \\
\hline$g$ & Transformation capacity at conversion plant \\
\hline$l$ & Final product demand location \\
\hline
\end{tabular}




\section{Parameters}

\begin{tabular}{|c|c|}
\hline RMAvailavility $_{i, n}$ & Available amount of raw materials type $\mathrm{n}$ at supplier location $\mathrm{i}$. \\
\hline $\operatorname{CapPP}_{j, c, f}$ & $\begin{array}{l}\text { Transformation capacity in tons of incoming materials at pretreatment located at } \mathrm{j} \text {, with } \\
\text { transformation technology } \mathrm{c} \text { and production capacity } \mathrm{f}\end{array}$ \\
\hline $\operatorname{CapW}_{k, d, g}$ & $\begin{array}{l}\text { Transformation capacity in tons of incoming materials at main production plants located at } \mathrm{k} \text {, with } \\
\text { transformation technology } \mathrm{d} \text { and production capacity } \mathrm{g}\end{array}$ \\
\hline$\alpha_{n, c, b}$ & Transformation rate of raw materials type $\mathrm{n}$ to intermediate products type $\mathrm{b}$ through technology $\mathrm{c}$. \\
\hline$\alpha_{b, d, a}$ & Transformation rate of intermediate products type $b$ to final products type a through technology $\mathrm{d}$. \\
\hline $\operatorname{Dem}_{m, b}(\operatorname{Max})$ & Amount of intermediate product type $\mathrm{b}$ demanded at client location $\mathrm{m}$ \\
\hline $\operatorname{Dem}_{l, a}(\operatorname{Max})$ & Amount of final product type a demanded at client location l \\
\hline $\operatorname{Prix}_{b}, \operatorname{Prix}_{a}$ & Market value for intermediate products type $b$ and final products type $a$ \\
\hline$y$ & Years for project evaluation \\
\hline DiscountRate & Value of discount rate for biorefinery projects \\
\hline$v_{a l}, v_{b a l_{a, k}}$ & $\begin{array}{l}\text { Value of reuse the intermediate products type } b \text { in the plant where they were produced at } j \text { and the } \\
\text { final products type } a \text { in the plants where they were produced at } k\end{array}$ \\
\hline $\begin{array}{l}\operatorname{TCost}_{i, j}, T \operatorname{Cost}_{j, k} \\
\operatorname{TCost}_{k, l}, T \operatorname{TCost} \\
j, m\end{array}$ & Transport cost for moving the materials between two points. \\
\hline$R_{M} \operatorname{Cost}_{n}$ & Market value of the raw material type $n$ \\
\hline$P \operatorname{Cost}_{n, j, c, f}=C_{0, n, j, c, f}$ & $\begin{array}{l}\text { Operation cost at pretreatment plants, depending on the raw material type } n \text {, the transformation } \\
\text { technology used } c \text {, the production capacity } f \text { and the plant location } j \text { (initial production cost) }\end{array}$ \\
\hline$P \operatorname{Cost}_{b, k, d, g}=C_{0, b, k, d, g}$ & $\begin{array}{l}\text { Operation cost at main production plants, depending on the intermediate product type } b \text {, the } \\
\text { transformation technology used } d \text {, the production capacity } g \text { and the plant location } k \text { (initial } \\
\text { production cost) }\end{array}$ \\
\hline$\beta_{c}, \beta_{d}$ & Factor related to learning for each transformation technology \\
\hline$\phi_{x, n, c, f}, \phi_{x, b, d, g}$ & $\begin{array}{l}\text { Rates of atmospheric pollution type } x \text { produced when transforming the raw material type } n \text { with } \\
\text { technology } c \text { and production capacity } f \text { at pretreatment plants (t pollution/t raw material) and when } \\
\text { transforming intermediate products type } b \text { with technology } d \text { and production capacity } g \text { at principal } \\
\text { plants (Ton pollution/Ton intermediate products) }\end{array}$ \\
\hline $\operatorname{PolCost}_{x}$ & Cost related to atmospheric pollution type $x$ production (USD/t pollution) \\
\hline$\psi_{y, n, c, f}, \psi_{y, b, d, g}$ & $\begin{array}{l}\text { Rates of residual water pollution type } y \text { produced when transforming raw material type } n \text { with } \\
\text { technology } c \text { and production capacity } f \text { at pretreatment plants (t pollution/t raw material) and when } \\
\text { transforming intermediate product type } b \text { with technology } d \text { and production capacity } g \text { at principal } \\
\text { plants (Ton pollution/Ton intermediate product). }\end{array}$ \\
\hline PolCostW & Cost related to residual water type $y$ production (USD/t pollution) \\
\hline$\omega_{z, n, c, f}, \omega_{z, b, d, g}$ & $\begin{array}{l}\text { Rates of solid waste type } z \text { generated when transforming the raw material type } n \text { with technology } c \\
\text { and production capacity } f \text { at pretreatment plants (Ton pollution/Ton raw material) and when } \\
\text { transforming intermediate products type } b \text { with technology } d \text { and production capacity } g \text { at } \\
\text { principal plants (Ton pollution/Ton intermediate products). }\end{array}$ \\
\hline PolCost $_{z}$ & Cost related to solid waste type $z$ production (USD/t pollution) \\
\hline $\operatorname{In} v_{k, d, g}, \operatorname{In} v_{j, c, f}$ & $\begin{array}{l}\text { Investment cost for implementing the production technology } d \text { in location } k \text { with the capacity } g \text { for } \\
\text { principal plants and implementing the technology } c \text { in location } j \text { with the capacity } f \text { for } \\
\text { pretreatment plants. }\end{array}$ \\
\hline $\operatorname{TaxRed}_{b}, \operatorname{Tax}_{\operatorname{Red}}$ & Tax exemption in USD per ton of sold bioproduct type $b$ and $a$ \\
\hline Certificate $_{i, n}$ & 1 If the supplier in the location $i$ is certified in the production of the raw material type $n, 0$ otherwise \\
\hline WRawMat $_{n}$ & Amount of water needed to produce raw materials type $n$ \\
\hline WPret $_{n, c, f}$, WPlant $_{b, d, g}$ & $\begin{array}{l}\text { Water needed to transform raw material } n \text { with technology } c \text { and production capacity } f \text { at } \\
\text { pretreatment and to transform the intermediate product } b \text { with technology } d \text { and production } \\
\text { capacity } g \text { at the principal production plants }\end{array}$ \\
\hline $\begin{array}{l}\text { Conf Land } \\
\text { ConfLand }_{j} \\
\text { Conf Land }_{k}\end{array}$ & $\begin{array}{l}1 \text { if supplier location at } i \text {, or the pretreatment plant at } j \text {, or the production plant at } k \text { has land tenure } \\
\text { conflicts }\end{array}$ \\
\hline$G I N I_{i}, G I N I_{j}, G I N I_{k}$ & $\begin{array}{l}\text { GINI index for land ownership in supplier location } i \text {, pretreatment plant location } j \text { and principal } \\
\text { production plant } k\end{array}$ \\
\hline $\begin{array}{l}\operatorname{DWork}_{R M, i, n} \\
\operatorname{IWork}_{R M, i, n}\end{array}$ & $\begin{array}{l}\text { Direct and indirect workers required to obtain } 1 \text { Ton of raw material type } n \text { at source location } i \text {, } \\
\text { respectively }\end{array}$ \\
\hline $\begin{array}{l}\text { DWork }_{\text {Pret }, j, c}, \\
\text { IWork } \\
\text { Pret,c,f }\end{array}$ & $\begin{array}{l}\text { Direct and indirect workers required to operate the pretreatment plants installed with technology } c \\
\text { and capacity } f\end{array}$ \\
\hline
\end{tabular}




\begin{tabular}{|c|c|}
\hline $\begin{array}{l}\text { DWork } \\
\text { IWrinc,d,g } \\
\text { IWrk } \\
\text { Princ,d,g }\end{array}$ & $\begin{array}{l}\text { Direct and indirect workers required to operate the principal plants installed with technology } d \text { and } \\
\text { capacity } g\end{array}$ \\
\hline $\operatorname{GasEm}_{\rho, a}$, GasEm $_{\rho, b}$ & $\begin{array}{l}\text { Gas emission type } \rho \text { (nitrogen oxide, particulate matter, carbon monoxide, hydrocarbons, Sulphur } \\
\text { dioxide equivalent and persistent organic pollutants) generated by the consumption of final products } \\
\text { type } a \text { and intermediate products type } b\end{array}$ \\
\hline $\begin{array}{l}R W a t P P_{n, c, f} \\
\text { RWatW }_{b, d, g}\end{array}$ & $\begin{array}{l}\text { Water reuse when transforming entering materials ( } n \text { and } b \text { ) with technologies } c \text { and } d \text {, and capacities } \\
f \text { and } g\end{array}$ \\
\hline$\vartheta_{\tau, i, n}$ & $\begin{array}{l}\text { Water pollution type } \tau \text { (phosphorus and nitrogen discharges generated by the raw material cultivation } \\
\text { type } n \text { at location } i \text {. The water pollution types } \tau \text { are. }\end{array}$ \\
\hline$S W_{n, c, f}, S W_{b, d, g}$ & $\begin{array}{l}\text { Wastewater generation when transforming the raw material type } n \text { with technology } c \text { and production } \\
\text { capacity } f \text { at pretreatment plants (Ton wastewater/Ton raw material) and transforming intermediate } \\
\text { products type } b \text { with technology } d \text { and production capacity } g \text { at principal plants (Ton wastewater } \\
\text { /Ton intermediate products) }\end{array}$ \\
\hline $\begin{array}{l}W s R e c P_{z, c, f} \\
W_{S R e c} W_{z, d, g}\end{array}$ & $\begin{array}{l}\text { Waste recover ratio by waste type } z \text { at pretreatment plant and at principal production plants } \\
\text { depending on production technology and capacity }\end{array}$ \\
\hline$\varepsilon_{\zeta, n}$ & $\begin{array}{l}\text { The soil deterioration rate due } \varsigma \text {, which is generated by the raw material cultivation type } n \text {. The } \\
\text { causes of soil deterioration } \varsigma \text { are total organic carbon, nitrogen, extractable phosphorus and } \\
\text { agrochemicals. }\end{array}$ \\
\hline $\mathrm{CO}_{2, n}$ & the equivalent $\mathrm{CO}_{2}$ generated at raw material type $n$ production at $i$ \\
\hline $\mathrm{CO}_{2 n, c, f}, \mathrm{CO}_{2}, d, g$ & $\begin{array}{l}\text { the generation rates of equivalent } \mathrm{CO}_{2} \text { at pretreatment plants and principal plants due entering } \\
\text { materials transformation, depending in technology and capacity production }\end{array}$ \\
\hline $\mathrm{CO}_{2 a}, \mathrm{CO}_{2 b}$ & The generation rates of equivalent $\mathrm{CO}_{2}$ due biorefinery products consumption. \\
\hline $\begin{array}{l}\mathrm{CO}_{2 i, n, j}, \mathrm{CO}_{2 j, b, k} \\
\mathrm{CO}_{2, a, l}, \mathrm{CO}_{2 j, b, m}\end{array}$ & The generation rates of equivalent $\mathrm{CO}_{2}$ due to logistic activities. \\
\hline RMYield & the raw material yield by hectare by type of raw material $n$ and location $i$ \\
\hline$R F_{i, n}, N R F_{i, n}$ & $\begin{array}{l}\text { Renewable fuel and non-renewable fuel required at raw material location for its production, by raw } \\
\text { material type } n \text { and location } i\end{array}$ \\
\hline $\begin{array}{l}\text { RFPret }_{n, c, f} \\
\text { RFPrinc }_{b, d, g} \\
\text { NRFPret }_{n, c, f} \\
\left.\text { NRFPrinc }_{b, d, g}\right)\end{array}$ & $\begin{array}{l}\text { Renewable fuel and non-renewable fuel used at pretreatment and principal plants for transformation } \\
\text { procedures, depending on raw material processed, production technology used and the production } \\
\text { capacity installed }\end{array}$ \\
\hline 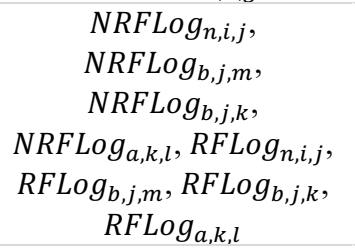 & Renewable fuel and non-renewable fuel used to transport the products between locations \\
\hline$\theta_{n}$ & Energy content of the raw material type $n$, in "MJ / Ton of raw material" \\
\hline$\delta$ & $\begin{array}{l}\text { Energy expenditure to transport the products, this factor is in "MJ/(Ton transported * } \\
\text { Km traveled)" }\end{array}$ \\
\hline Dist $_{i, j}$ & Distance in $K m$ between supplier $i$ and pretreatment plant $j$ \\
\hline Dist $_{j, k}$ & Distance in $K m$ between the pretreatment plant $j$ and the main production plant $k$ \\
\hline Dist $_{k, l}$ & Distance in $K m$ between the main production plant $k$ and the customer located in $l$ \\
\hline Dist $_{j, m}$ & Distance in $K m$ between the pretreatment plant $j$ and the customer located in $m$ \\
\hline$\beta_{n, c, f}$ & $\begin{array}{l}\text { Energy consumption to transform incoming raw material type } n \text { with technology } c \text { and production } \\
\text { capacity } f \text { (If this value is independent of production capacity, it means that economies of scale are } \\
\text { not considered in the production pretreatment plant) }\end{array}$ \\
\hline$\beta_{b, d, g}$ & $\begin{array}{l}\text { Energy consumption to transform the incoming intermediate products type } b \text { with the technology } d \\
\text { and the production capacity } g \text { (If this value is independent of the production capacity, it means that } \\
\text { they are not considered economies of scale in the main production plants) }\end{array}$ \\
\hline$\theta_{b}, \theta_{a}$ & $\begin{array}{l}\text { Energy content of intermediate product type } b \text {, in "MJ / Ton of intermediate product"; and final } \\
\text { product type } a \text {, in "MJ / Ton of final product" }\end{array}$ \\
\hline $\operatorname{Rec} R M_{i, n}$ & 1 if the raw material type $n$ from supplier $i$ is recycled, 0 otherwise \\
\hline LimBudget & Maximum budget available for biorefinery projects related to plant installation \\
\hline $\operatorname{incCap}_{j}, \operatorname{incCap}_{k}$ & $\begin{array}{l}\text { Allowable incentive cap for pretreatment plants located at } j \text { and for main production plants located } \\
\text { at } k\end{array}$ \\
\hline $\operatorname{incPer}_{j}$, incPerW $_{k}$ & $\begin{array}{l}\text { Cap percentage of the total construction cost for pretreatment plant } j \text { to be subsidized and for main } \\
\text { production plant } k \text { to be subsidized }\end{array}$ \\
\hline BudProjValueTech & Budgetary limit related to the project value to develop technologies \\
\hline
\end{tabular}




\begin{tabular}{cl}
\hline Dem $_{a_{E}, l}$ & Biobased electricity demand \\
$\xi \xi$ & $\begin{array}{l}\text { Rate that represent the increase in electricity demand by USD of governmental investment in } \\
\text { electricity infrastructure }\end{array}$ \\
\hline TotalWaterAval & Total available water \\
\hline
\end{tabular}

\section{References}

Awudu I, Zhang J. Uncertainties and sustainability concepts in biofuel supply chain management: A review. Renew Sustain Energy Rev 2012;16:1359-68. doi:10.1016/j.rser.2011.10.016.

Bai Y, Ouyang Y, Pang J-S. Biofuel supply chain design under competitive agricultural land use and feedstock market equilibrium. Energy Econ 2012;34:1623-33. doi:10.1016/j.eneco.2012.01.003.

Barbosa-Póvoa AP, da Silva C, Carvalho A. Opportunities and challenges in sustainable supply chain: An operations research perspective. Eur J Oper Res 2018;268:399-431. doi:10.1016/j.ejor.2017.10.036.

Basto Aluja M del P. SIMULACIÓN Y EVALUACIÓN ECONÓMICA DEL PROCESO DE PRODUCCIÓN DE BIODIÉSEL A PARTIR DEL ACEITE DE PALMA, EMPLEANDO REACTORES DE PELÍCULA LÍQUIDA-LÍQUIDA OPERADOS EN CONTRACORRIENTE Y CO-CORRIENTE. Universidad Nacional de Colombia Facultad de Ingeniería, 2016.

Bautista Rodríguez SC. Sustainability assessment of biodiesel production in Colombia. 2015.

Bautista S, Narvaez P, Camargo M, Chery O, Morel L. Biodiesel-TBL+: A new hierarchical sustainability assessment framework of PC\&amp;I for biodiesel production - Part I. Ecol Indic 2016;60:84-107. doi:10.1016/j.ecolind.2015.06.020.

Black MJ, Sadhukhan J, Day K, Drage G, Murphy RJ. Developing database criteria for the assessment of biomass supply chains for biorefinery development. Chem Eng Res Des 2016;107:253-62. doi:10.1016/j.cherd.2015.10.046.

Blasco X, Herrero JM, Reynoso-Meza G, Iranzo MAM. Interactive tool for analyzing multiobjective optimization results with Level Diagrams. GECCO 2017 - Proc Genet Evol Comput Conf Companion 2017:1689-96. doi:10.1145/3067695.3082553.

Bueno L, Toro C, Martín M. Techno-economic evaluation of the production of polyesters from glycerol and adipic acid. Chem Eng Res Des 2015;93:432-40.

doi:http://dx.doi.org/10.1016/j.cherd.2014.05.010.

Bueno L, Toro C, Martín M. Scale-up and Techno-economical study for the production of polyesters from glycerol. In: Klemes JJ, Varbanov PS, Yen Liew P, editors. 24th Eur. Symp. Comput. Aided Process Eng., Budapest, Hungary: Elsevier; 2014, p. 43-8.

Cambridge Dictionaries Online. Self-sustaining - Business English Dictionary 2015. http://dictionary.cambridge.org/fr/dictionnaire/anglais-des-affaires/self-sustaining (accessed June 22, 2015).

Ceapraz IL, Kotbi G, Sauvee L. The territorial biorefinery as a new business model. Bio-Based Appl Econ 2016;5:47-62. doi:10.13128/BAE-15379.

Chopra S, Meindl P. Supply Chain Management. 5 edition. Boston: Prentice Hall; 2012.

Costa Y, Duarte A, Sarache W. A decisional simulation-optimization framework for sustainable facility location of a biodiesel plant in Colombia. J Clean Prod 2017. doi:10.1016/j.jclepro.2017.08.126.

DANE DAN de E. Encuesta Anual Manufacturera. Sistema de Consulta. Encuesta Anu Manuf 2017. http://formularios.dane.gov.co/encuestas/eam/seleccion.htm? $\mathrm{x}=33 \& \mathrm{y}=22$ (accessed June 29, 2017).

Ekşioğlu SD, Acharya A, Leightley LE, Arora S. Analyzing the design and management of 
biomass-to-biorefinery supply chain. Comput Ind Eng 2009;57:1342-52.

doi:10.1016/j.cie.2009.07.003.

Espinoza Pérez AT. Biorefinery supply chain design optimization under sustainability dimensions. Université de Lorraine, 2017.

Espinoza Pérez AT, Bautista S, Narvaez PC, Alfaro M, Camargo M. Sustainability assessment to support governmental biodiesel policy in Colombia: A system dynamics model. J Clean Prod 2017a;141:1145-63. doi:10.1016/j.jclepro.2016.09.168.

Espinoza Pérez AT, Camargo M, Narváez Rincón PC, Alfaro Marchant M. Key challenges and requirements for sustainable and industrialized biorefinery supply chain design and management: A bibliographic analysis. Renew Sustain Energy Rev 2017b;69:350-9. doi:http://dx.doi.org/10.1016/j.rser.2016.11.084.

Fedebiocombustibles (Federación Nacional de Biocombustibles de Colombia). Producción y Venta de Biodiesel 2017. http://www.fedebiocombustibles.com/estadistica-produccion-tituloBiodiesel.htm (accessed April 1, 2018).

Fedebiocombustibles FN de B de C. Federación Nacional de Biocombustibles de Colombia 2015. http://www.fedebiocombustibles.com/v3/estadistica-mostrar_info-titulo-Biodiesel.htm (accessed March 6, 2015).

Fedepalma. SISPA Fedepalma 2016.

Fonseca CM, Fleming PJ. An Overview of Evolutionary Algorithms in Multiobjective Optimization. Evol Comput 1995;3:1-16. doi:10.1162/evco.1995.3.1.1.

Global Reporting Initiative. Consolidated set of GRI sustainability reporting standards. 2016.

Guillén G, Badell M, Espuña A, Puigjaner L. Simultaneous optimization of process operations and financial decisions to enhance the integrated planning/scheduling of chemical supply chains. Comput Chem Eng 2006;30:421-36. doi:10.1016/j.compchemeng.2005.10.015.

Hernández Castiblanco Á, Amórtegui Gómez IC. Estudio De Factibilidad Para El Establecimiento De Un Proyecto Productivo De Jatropha En Territorio Colombiano. Universidad Distrital Francisco José De Caldas Facultad Del Medio Ambiente Y Recurso Naturales Especialización En Gerencia De Recursos Naturales, 2015.

Hillier F, Lieberman G. Introduction to Operations Research. 7th ed. Pennsylvania State University: McGraw-Hill, 2001; 2001.

Höltinger S, Schmidt J, Schönhart M, Schmid E. A spatially explicit techno-economic assessment of green biorefinery concepts. Biofuels, Bioprod Biorefining 2014;8:325-41. doi:10.1002/bbb.1461.

Huband S, Hingston P, Barone L, While L. A review of multiobjective test problems and a scalable test problem toolkit. IEEE Trans Evol Comput 2006;10:477-506.

doi:10.1109/TEVC.2005.861417.

J.G.Cadavid, R.D.Godoy-Silva, P.C.Narvaez, M.Camargo, C.Fonteix. Biodiesel production in a counter-current reactive extraction column: Modelling, parametric identification and optimisation. Chem Eng J 2013;228:717-23. doi:https://doi.org/10.1016/j.cej.2013.05.040.

Majid Eskandarpour PD. Sustainable supply chain network design: An optimization-oriented review. Omega 2015;54. doi:10.1016/j.omega.2015.01.006.

De Meyer A, Cattrysse D, Rasinmäki J, Van Orshoven J. Methods to optimise the design and management of biomass-for-bioenergy supply chains: A review. Renew Sustain Energy Rev 2014;31:657-70. doi:10.1016/j.rser.2013.12.036.

Mortazavi A, Arshadi Khamseh A, Azimi P. Designing of an intelligent self-adaptive model for supply chain ordering management system. Eng Appl Artif Intell 2015;37:207-20.

doi:10.1016/j.engappai.2014.09.004.

Narváez PC, Sánchez FJ, Godoy-Silva RD. Continuous methanolysis of palm oil using a liquidliquid film reactor. J Am Oil Chem Soc 2009;86:343-352. doi:https://doi.org/10.1007/s11746009-1356-9. 
Narzisi G, Mysore V, Mishra B. Multi-objective evolutionary optimization of agent-based models: An application to emergency response planning, San Francisco, CA: 2006, p. 224-30.

Nguyen Q, Bowyer J, Howe J, Bratkovich S, Groot H, Pepke E, et al. Global production of second generation biofuels: trends and influences. 2017.

Özdenkçi K, De Blasio C, Muddassar HR, Melin K, Oinas P, Koskinen J, et al. A novel biorefinery integration concept for lignocellulosic biomass. Energy Convers Manag 2017;149:974-87. doi:10.1016/j.enconman.2017.04.034.

Rodríguez AG, Mondaini AO, Hitschfeld MA. Bioeconomía en América Latina. 2017.

Ronzon T, Lusser M, Klinkenberg (ed.) M, Landa L, Sanchez Lopez (ed.) J, M'Barek R, et al. Bioeconomy Report 2016. vol. EUR 28468. Brussels: 2017. doi:10.2760/20166.

Ruiz-mercado GJ, Martin W, King L, States U, Smith RL, Gonzalez MA. Sustainability Indicators for Chemical Processes : I . Taxonomy 2012:2309-28.

Sammons N, Eden M, Yuan W, Cullinan H, Aksoy B. A flexible framework for optimal biorefinery product allocation. Environ Prog 2007;26:349-54. doi:10.1002/ep.10227.

Shastri Y. Renewable energy, bioenergy. Curr Opin Chem Eng 2017;17:42-7. doi:10.1016/j.coche.2017.06.003.

Singh A, Chu Y, You F. Biorefinery Supply Chain Network Design under Competitive Feedstock Markets: An Agent-Based Simulation and Optimization Approach. Ind Eng Chem Res 2014;53:15111-26. doi:10.1021/ie5020519.

Suárez Palacios OY, Nárvaez Rincón PC, Corriou J-P. Production et modelisation de glycerolesters comme plastifiants pour le PVC. 2011.

Sukumara S, Faulkner W, Amundson J, Badurdeen F, Seay J. A multidisciplinary decision support tool for evaluating multiple biorefinery conversion technologies and supply chain performance. Clean Technol Environ Policy 2013;16:1027-44. doi:10.1007/s10098-013-07036.

Taha HA. Operations Research: An Introduction. Pearson; 9 edition (September 8, 2010); 2010. Tsagkari, M., Couturier, J., Kokossis, A., \& Dubois J. Early-Stage Capital Cost Estimation of Biorefinery Processes: A Comparative Study of Heuristic Techniques. ChemSusChem 2016;9:2284-97. doi:10.1002/cssc.201600309.

Tsolakis NK, Keramydas CA, Toka AK, Aidonis DA, Iakovou ET. Agrifood supply chain management: A comprehensive hierarchical decision-making framework and a critical taxonomy. Biosyst Eng 2014;120:47-64. doi:10.1016/j.biosystemseng.2013.10.014.

Valdivia M, Galan JL, Laffarga J, Ramos JL. Biofuels 2020: Biorefineries based on lignocellulosic materials. Microb Biotechnol 2016;9:585-94. doi:10.1111/1751-7915.12387.

de Wit M, Junginger M, Lensink S, Londo M, Faaij A. Competition between biofuels: Modeling technological learning and cost reductions over time. Biomass and Bioenergy 2010;34:203-17. doi:10.1016/j.biombioe.2009.07.012.

You F, Tao L, Graziano DJ, Snyder SW. Optimal design of sustainable cellulosic biofuel supply chains: Multiobjective optimization coupled with life cycle assessment and input-output analysis. AIChE J 2012;58:1157-80. doi:10.1002/aic.12637.

Yue D, Slivinsky M, Sumpter J, You F. Sustainable Design and Operation of Cellulosic Bioelectricity Supply Chain Networks with Life Cycle Economic, Environmental, and Social Optimization. Ind Eng Chem Res 2014a;53:4008-29. doi:10.1021/ie403882v.

Yue D, You F, Snyder SW. Biomass-to-bioenergy and biofuel supply chain optimization: Overview, key issues and challenges. Comput Chem Eng 2014b;66:36-56.

doi:10.1016/j.compchemeng.2013.11.016.

Zhou A, Qu B-Y, Li H, Zhao S-Z, Suganthan PN, Zhang Q. Multiobjective evolutionary algorithms: A survey of the state of the art. Swarm Evol Comput 2011;1:32-49.

doi:10.1016/j.swevo.2011.03.001. 
